Spin-up of a two-layer rotating stratified fluid in a variable-depth container

Hewitt, R.E. and Foster, M.R. and Davies, P.A.

2001

MIMS EPrint: 2013.64

Manchester Institute for Mathematical Sciences

School of Mathematics

The University of Manchester

\footnotetext{
Reports available from: http://eprints.maths.manchester.ac.uk/

And by contacting: The MIMS Secretary

School of Mathematics

The University of Manchester

Manchester, M13 9PL, UK
} 


\title{
Spin-up of a two-layer rotating stratified fluid in a variable-depth container
}

\author{
By R. E. HEWITT ${ }^{1,3}$, M. R. FOSTER ${ }^{2}$ AND P. A. DAVIES \\ ${ }^{1}$ Department of Mathematics, University of Manchester, Oxford Road, \\ Manchester M13 9PL, UK \\ ${ }^{2}$ Department of Aerospace Engineering and Aviation, The Ohio State University, \\ Columbus, OH 43210, USA \\ ${ }^{3}$ Department of Civil Engineering, The University, Dundee DD1 4HN, UK
}

(Received 18 August 1999 and in revised form 23 October 2000)

We consider the spin-up of a two-layer, stably (density) stratified fluid in a rotating container with an axisymmetric sloping base and cylindrical walls. Details of the spinup readjustment mechanisms are presented under the assumption of small impulsive changes in the rotation rate of the container. It is shown that the relative positions of the density interface and the discontinuity in wall slope determine the qualitative large-time spin-up response of the fluid. The density interface leads to a spin-up readjustment in each of the fluid layers that is essentially independent. However, when the density interface is below the boundary-slope discontinuity, a sub-region of the upper layer is predicted to readjust in an algebraic rather than exponential manner. A detailed sequence of laboratory experiments have been performed to confirm the predictions of the linear spin-up analysis.

\section{Introduction}

The processes of spin-up and spin-down in rotating fluid bodies have an established relevance to large-scale geo- and astrophysical flows (see, for example, the review by Benton \& Clark 1974) and a wider applicability to a number of industrial problems such as centrifugal separation and projectile stability (O'Donnell \& Linden 1991). The term spin-up (-down) usually refers to the transient response of a rotating fluid body to an instantaneous increase (decrease) in the rotation rate of the container, though there are other geophysically interesting flow configurations where the spin-up (-down) mechanism also operates. For example, in the case studied by Spence, Foster \& Davies 1992, a fluid body readjusts via a spin-up mechanism when the uniform rigid body rotation is perturbed by the sudden imposition of a constant surface stress (see also Pedlosky 1971).

For homogeneous fluids, the time taken for the system to adjust fully (that is, spin-up/down) to a new rotation rate, $\Omega \pm \Delta \Omega$, from an initial rate, $\Omega$, is known to be $E^{\prime-1 / 2} \Omega^{-1}$ (Greenspan \& Howard 1963); here $E^{\prime}$ is the Ekman number of the flow defined by $E^{\prime}=v / \Omega H^{2}$ while $v$ and $H$ are the kinematic viscosity and total depth of the fluid respectively. In this formulation, the result concerning the spin-up time scale is valid for $\Delta \Omega / \Omega \ll 1$, though experiments and theoretical analyses by Weidman $(1976 a, b)$ have also investigated the effects of nonlinear $(\Delta \Omega / \Omega=O(1))$ spin-up. In all cases, the mechanism involved is stretching (compression) of the vortex lines of the rigid rotation by pumping of fluid into (or out of) the Ekman boundary layers on the 
horizontal surfaces of the container (Greenspan \& Howard 1963; Wedemeyer 1964; Greenspan \& Weinbaum 1965). Diffusive effects have no real part in this adjustment process. $\dagger$

If the rotating fluid under consideration is also stably density stratified, the transient adjustment is very much slower, occurring in two stages (see Pedlosky 1967; Walin 1969; Sakurai 1969; Beardsley et al. 1979; Buzyna \& Veronis 1971). There is an initial adjustment (occurring on the same $E^{\prime-1 / 2}$ time scale as the homogeneous spin-up) to a quasi-steady state in which the interior horizontal velocity has a linear vertical shear. However, the final adjustment to a new, steady, equilibrium state occurs much more slowly over the diffusive time scale $E^{\prime-1}$ (see also Spence et al. 1992). Laboratory experiments by Buzyna \& Veronis (1971) have confirmed many of the features of these analytical predictions.

For two-layer immiscible stratified fluids with upper and lower layers of depth $H_{u}$ and $H_{l}$ respectively, spin-up is achieved separately on $E_{u, l}^{\prime-1 / 2}$ time scales appropriate to the individual layers (with $E_{u, l}$ defined using $H_{u, l}$ as the appropriate length scale and kinematic viscosities of $v_{u}, v_{l}$ ). If either or both of the ratios of depths and viscosities differ significantly from unity, the consequent differences in spin-up times between the upper and lower layers results in a dome-like deformation of the interface during the adjustment process (see, for example, Kim \& Hyun 1994).

In the present study we investigate the transient response of a two-layer rotating fluid to a change in the background rotation rate of the container, for a case in which the two homogeneous layers are separated by a thin, high- $N$ region (where $N$ is the Brunt-Väisälä frequency, which we define in detail later). Attention is focused on containers for which the fluid depth varies linearly with radius. We search in particular for the effects of the sloping bottom boundary in the transient response of the fluid. The response mechanism for cases in which the interface intersects the sloping base is compared with those in which the interface is either remote from the lower boundary or below the slope discontinuity (see figure 1 below). Laboratory experiments are described in which velocity-field data and density changes have been measured over a range of parameter values.

Compared with the cases discussed above, much less is known about transient adjustments of rotating homogeneous and stratified fluid systems in variable-depth containers of the type investigated here. Before turning to these cases, we note that even steady-state motions in such geometries are not fully examined in the literature. Hide \& Hocking (1979) and Page (1982) showed that a homogeneous, steady flow in a container with a planar bottom cutting obliquely across the cylinder forms a boundary current, a sidewall shear layer whose structure is quite different from counterpart layers in constant-depth containers (see Stewartson 1966, for example). Van Heijst, Maas \& Williams (1994) have considered the rather complex case of spinup in a non-axisymmetric container (a rectangular channel) having a sloping base, and have demonstrated clearly that the fluid in the shallow part of the tank spins up much more quickly than the deeper fluid. For fluids that are stratified with a solute (e.g. salt in water), Phillips (1970) and Wunsch (1970) have found that the slope itself drives a weak diffusive current along it with no other boundary forcing, although in a saltstratified medium the associated slope-velocity Reynolds number is expected (Phillips $1970)$ to be very small $\left(10^{-3}\right)$. In a closed container, this slope current eventually decays to rest, with a very narrow layer adjacent to the lower wall being well-mixed as a result.

$\dagger$ Since the spin-up and spin-down processes are identical for the linear case, a distinction between the two is no longer made in this paper. 

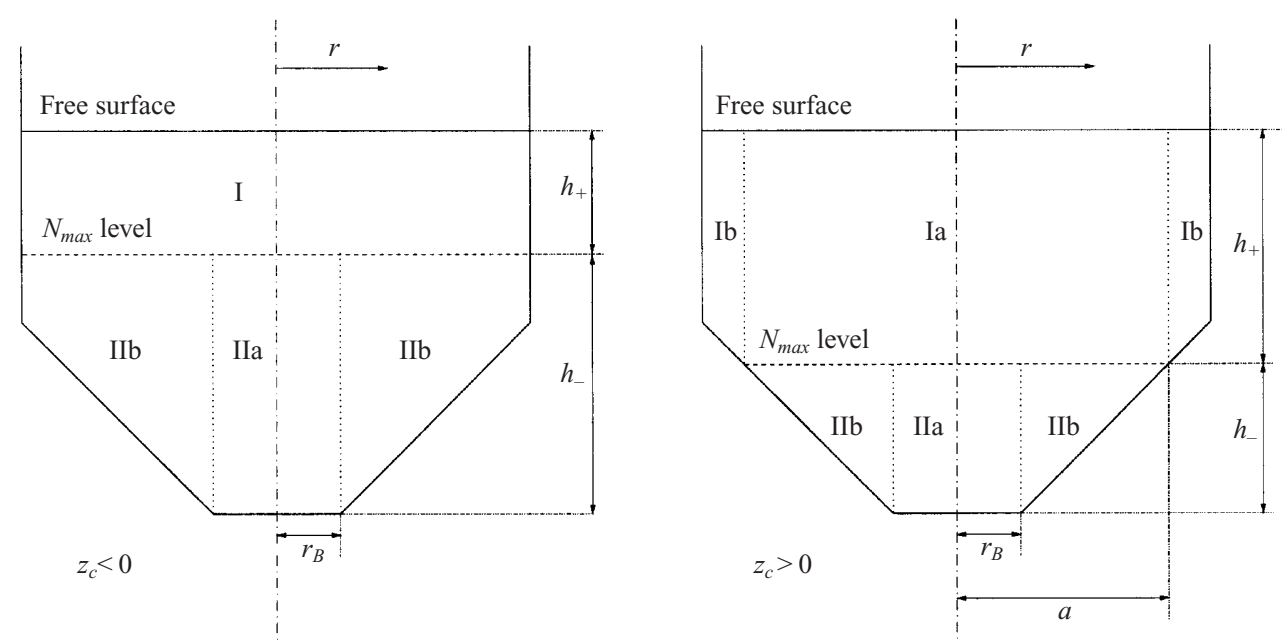

FiguRE 1. The interior flow regions described by the linear spin-up analysis. The non-dimensional extent of each region is as denoted, with the cylinder wall at $r=1$. Note that the container utilized in the laboratory experiments is such that $r_{B} \ll 1 . z$ is measured relative to the location defined by $N=N_{\max }$, as defined in the text. The quantity $z_{c}$ is the relative vertical location of the cone/cylinder interface to the mid-level of the density interface.

The recent work of Duck, Foster \& Hewitt (1997); Hewitt et al. (1999a) and Hewitt, Duck \& Foster (1999b) has considered the nonlinear spin-up (and spin-down) of a fluid that is linearly stratified with a dissolved solute in container geometries with sloping boundaries. The work extended the previous linear analyses of MacCready \& Rhines (1991) and Thorpe (1987) by considering the nature of the boundary layer when the changes in rotation are of the same order as the initial/final rotation rate. A combination of computational approaches, asymptotic analysis and laboratory experiments has shown that the presence of both rotation and buoyancy effects can lead to a rich variety of boundary-layer behaviours for nonlinear spin-up/down.

Thus, in this paper we shall consider the global linear spin-up of a two-layer fluid in a variable-depth container, in which two homogeneous regions of fluid are separated by a thin, high- $N$ layer, rather than two immiscible fluid layers. In $\S 2$, we provide the theoretical formulation and present some analytical results. Section 2.1 discusses the spin-up mechanism in a circular cylinder for a two-layer fluid and this analysis is extended in $\S 2.2, \S 2.3$ and $\S 2.4$ for the case of a circular cylinder with a conical base. In $\S 3$, the theoretical predictions are summarized and $\S 4$ provides the details of a corresponding laboratory investigation of the spin-up readjustment. The results of the experimental work and comparisons to the theoretical predictions are given in $\S 5$. A brief qualitative description of the first observable nonlinear effects is given in $\S 5.3$, and conclusions are given in $\S 6$.

\section{Formulation and theoretical results}

Figure 1 shows the configuration under consideration. A cylindrical container of radius $R$, with a conical lower surface, is rotating initially with uniform angular velocity $\Omega$ about the vertical $z$-axis. The container is filled with two miscible fluids of upper and lower layer densities $\rho_{o}$ and $\rho_{o}+\Delta \rho$ and depths $H_{u}$ and $H_{l}$ respectively, in hydrostatic equilibrium and solid body rotation. The layers are separated by an interfacial layer of thickness $R \delta$, within which the density $\rho(z)$ varies smoothly 
and monotonically with depth $z$ and in which the local buoyancy frequency $N(z)$ has a maximum value $N_{\max }$ midway between the upper and lower 'surfaces' of the interfacial layer. Here, $N(z)$ is defined by $N^{2}=-\left(g / \rho_{o}\right) \partial \rho / \partial z$, where $g$ is the gravitational acceleration. Attention is restricted to cases in which the difference in kinematic viscosity between the two constituent fluids is negligible. The upper surface of the system is free and the lower boundary consists of a conical solid surface of base angle $\alpha$, cut by a horizontal plate of radius $R r_{B}$. Throughout the analysis, we use a circular polar coordinate system $(r, \theta, z)$, with velocity components $(u, v, w)$ in those directions. For later convenience, we fix the coordinate system so that $z=0$ in the centre of the density interface, that is, where $N$ is a maximum.

At time $t=0$ the rotation rate of the system is changed by $\Delta \Omega$ and the system responds to this change in a manner that is conveniently described in terms of the individual values of the following set of dimensionless dynamical parameters:

$$
\begin{gathered}
R o=\frac{\Delta \Omega}{\Omega}, \quad \text { a Rossby number, } \\
B u=\frac{N_{\max }^{2}}{\Omega^{2}}, \quad \text { a Burger number, } \\
E=\frac{v}{\Omega R^{2}}, \quad \text { an Ekman number, }
\end{gathered}
$$

and the geometrical ratios $r_{B}, \delta$ (described below), $h_{+}=H_{u} / R, h_{-}=H_{l} / R$, and the angle $\alpha$.

Since the motion is driven by a differential rotation, all dynamical dependent variables will scale with $\Delta \Omega$. We therefore write the physical dependent variables, marked with a $\left(^{*}\right)$, as

$$
\begin{gathered}
\boldsymbol{u}^{*}=\Delta \Omega R \boldsymbol{u}, \\
p^{*}=\rho_{R} \Omega \Delta \Omega R^{2} p+p_{o}^{*}(z), \\
\rho^{*}=\rho_{R}+\rho_{o}(z)+\frac{\rho_{R} \Omega \Delta \Omega R}{g} \rho, \\
N^{2}=-g \frac{\rho_{o}^{\prime}(z)}{\rho_{R}},
\end{gathered}
$$

where the quantity $p_{0}^{*}(z)$ is the hydrostatic pressure corresponding to the density distribution $\rho_{o}(z)$ and $R$ is the container's maximum radius. In the following formulation, we scale spatial variables with $R$, so $\left(r^{*}, z^{*}\right)=(R r, R z)$, and we suppose that the Rossby number $R o$ is small. In the experiments the Brunt frequency is sharply peaked near the initial undisturbed interface; we model this by writing

$$
N^{2}=\Omega^{2} \frac{S}{\delta^{2}} \exp \left(-(z / \delta)^{2}\right), \quad \text { where } \quad E^{1 / 2} \ll \delta \ll E^{1 / 4},
$$

and the Burger number $S$ employed in the analysis is related to the usual Burger number $B u$ above by $S=B u \delta^{2}$; it is taken to be $O(1)$. The origin of the restrictions on $\delta$ are discussed in detail in $\S 2.4$.

The continuity, Boussinesq-approximated Navier-Stokes and density transport equations become (in a rotating frame)

$$
\begin{gathered}
\nabla \cdot \boldsymbol{u}=0, \\
E^{1 / 2} \frac{\partial \boldsymbol{u}}{\partial t}+2 \boldsymbol{k}_{\wedge} \boldsymbol{u}+\nabla p=E \nabla^{2} \boldsymbol{u}-\rho \boldsymbol{k},
\end{gathered}
$$




$$
\begin{aligned}
& \text { Spin-up of a two-layer rotating stratified fluid } \\
& \qquad E^{1 / 2} \frac{\partial \rho}{\partial t}-\frac{S}{\delta^{2}} \exp \left(-(z / \delta)^{2}\right) w=\frac{E}{\sigma} \nabla^{2} \rho,
\end{aligned}
$$

where the nonlinear terms have been omitted. The Ekman number $E$ is most conveniently based on the radius $R$ of the apparatus; $\sigma$ is the Schmidt number and we have taken the time scale to be that appropriate to the spin-up of a homogeneous fluid unless otherwise noted, so $t^{*}=R /(v \Omega)^{1 / 2} t$. We wish to construct an asymptotic solution to these equations with appropriate boundary conditions, namely

$$
\begin{gathered}
\boldsymbol{u}=\boldsymbol{k}_{\wedge} \boldsymbol{r} \equiv \boldsymbol{u}_{B} \quad \text { at all solid boundaries, } \\
\frac{\partial \rho}{\partial n}=0 \quad \text { at all solid boundaries, } \\
\text { stress continuity at } z=h_{+} .
\end{gathered}
$$

Initial conditions require that the velocity field be zero at $t=0$. (Note that from (2.8) we have taken the vertical coordinate, $z$, to have its origin at the level of the peak value of $N$.)

Because the initial $z$-dependent density gradient cannot satisfy the stated condition on $\rho$ in the $\delta$-region of the sloping walls, there will be thin, unsteady, diffusive layers on those walls. However, these layers have thickness $R E^{1 / 2}(\delta / S \sigma)^{1 / 4}$, developing over a time scale $(\sigma \delta / S)^{1 / 2} / \Omega$ and so have little bearing on this analysis so long as the Schmidt number, $\sigma$, is large and $\delta$ is restricted as indicated in equation (2.8). In typical laboratory experiments involving saline solutions, the relevant Schmidt number is $\sigma \sim 10^{3}$.

It is well known that on horizontal boundaries equations (2.9)-(2.11) admit Ekman layers whose structure leads to a compatibility condition at the boundary which may be applied to geostrophic solutions in lieu of repeated boundary-layer analysis. This compatibility condition in our notation is

$$
w=-\frac{E^{1 / 2}}{2} \frac{1}{r} \frac{\partial}{\partial r}\left[r\left(v-v_{B}\right)\right], \quad \text { at } \quad z=-h_{-},
$$

where the $B$ subscript denotes velocities at the boundary. This same condition must be applied on the sloped portion of the lower wall, where it takes the form

$$
w=u \tan \alpha+\frac{E^{1 / 2}}{2 \sqrt{\cos \alpha}} \frac{1}{r} \frac{\partial}{\partial r}\left[r\left(v-v_{B}\right)\right] .
$$

This formula has been specialized for axisymmetry, which we shall assume throughout this discussion. In the subsequent analysis, we take $v_{B}=r$ for rigid rotation.

The nature of the flow in the (thin) interfacial layer, centred at $z=0$ and having width $\delta$ is a question of considerable complexity, and its discussion is deferred until $\S 2.4$. For the moment, we anticipate the crucial piece of information from that analysis, namely that, insofar as the outer flow is concerned, the layer at $z=0$ acts as a non-penetrable barrier to the flow above and below it. Based on analysis for situations with two immiscible fluids, one might expect Ekman layers on either side of the interface, but they do not occur here; a further discussion of this point is given in $\S 2.4$.

Hence, we obtain

$$
w=0 \quad \text { at } \quad z=0
$$

to leading order in $E$. Thus, the stratification is so large in this thin $\delta$-zone that vertical motion is inhibited, thereby isolating Region I from Region II (see figure 1) in all that follows. 


\subsection{Spin-up in a cylindrical container}

Before examining the spin-up in a cylinder-cone container, we turn first to a simpler problem that is instructive: spin-up in a cylindrical container. Referring to figure 1, we note that the upper (free) and lower horizontal surfaces of the container are given by $z=h_{+}$and $z=-h_{-}$respectively. From (2.17) above, we require that $w$ must vanish at $z=0$ at least to order $E^{1 / 2}$. Since there can be no Ekman layer beneath the upper (free) boundary, the usual homogeneous-fluid spin-up mechanism involving Ekman pumping cannot be active in the upper layer, Region I. In the lower layer (Region II) the asymptotic expansion proceeds in powers of $E^{1 / 2}$. The leading-order velocity is in the swirl direction only and geostrophic, so that $v_{0}=v_{0}(r, t)$ only. To next order, since there is no baroclinic density variation in Region II,

$$
\begin{gathered}
-2 v_{0}+\frac{\partial p_{0}}{\partial r}=0, \\
\frac{\partial p_{0}}{\partial z}=0, \\
\frac{\partial v_{0}}{\partial t}+2 u_{1}=0, \\
\frac{1}{r} \frac{\partial r u_{1}}{\partial r}+\frac{\partial w_{1}}{\partial z}=0 .
\end{gathered}
$$

Utilizing the Ekman suction condition (2.15) to determine the rate of change of $w_{1}$ with $z$ leads to a partial differential equation for the vorticity in the flow beneath the interfacial layer,

$$
\frac{\partial Z}{\partial t}+\frac{1}{h_{-}}\left(Z-Z_{B}\right)=0, \quad Z \equiv \frac{1}{r} \frac{\partial r v_{0}}{\partial r},
$$

whose solution for the velocity is the familiar homogeneous spin-up solution given first by Greenspan \& Howard (1963),

$$
v_{0}=v_{B}\left(1-\mathrm{e}^{-t / h_{-}}\right) .
$$

For the flow in Region I (the upper layer), since there is no Ekman pumping the usual spin-up time scale is not relevant. The only relevant scale is the viscous diffusion time, $a^{2} / v$, and so the leading-order term in the $E^{1 / 2}$ expansion in Region I gives the partial differential equation

$$
\frac{\partial^{2} v_{0}}{\partial r^{2}}+\frac{\partial v_{0}}{\partial r}-\frac{v_{0}}{r^{2}}=\frac{\partial v_{0}}{\partial t^{\prime}},
$$

where $t^{\prime}=t E^{1 / 2}$. The solution is therefore

$$
\begin{gathered}
v_{0}=v_{B}\left[1-\sum_{n=1}^{\infty} a_{n} J_{1}\left(j_{n} r\right) \exp \left(-j_{n}^{2} t^{\prime}\right)\right], \\
a_{n}=\left\{\int_{0}^{1} r J_{1}\left(j_{n} r\right) \mathrm{d} r\right\}\left\{\int_{0}^{1} r J_{1}^{2}\left(j_{n} r\right) \mathrm{d} r\right\}^{-1},
\end{gathered}
$$

where $j_{n}$ is the $n$th zero of $J_{1}(x)$.

2.2. Spin-up in the cone/cylinder for $z_{c}<0$ : interface intersects the vertical wall We now return to a cylindrical container with a conical base and consider the case in which the density interface is above the conical surface. This configuration is denoted 
by $z_{c}<0$ (see figure 1). The fluid above the interface (Region I) is wholly bounded by vertical sidewalls. Hence, the upper level of the fluid has a velocity $v_{0}$ that is given once again by equation (2.25); therefore, on the time scale under examination here, no spin-up has occurred except sufficiently close to the wall. It is the region below the interface that differs from the case considered in $\S 2.1$, since now the lower layer has a boundary that has a conical portion. In Region IIa, where $r<r_{B}$, the solution is given by equation (2.23).

In Region IIb, the flow is somewhat more complicated due to the fact that the lower wall is not horizontal. Equations (2.18)-(2.21), when utilized to eliminate $u_{1}$, lead to

$$
\frac{\partial}{\partial t}\left(\frac{1}{r} \frac{\partial r v_{0}}{\partial r}\right)=2 \frac{\partial w_{1}}{\partial z} .
$$

The Ekman condition (2.16) determines $w_{1}$ and use of (2.20) leads to an equation whose first integral is the equation satisfied by $v_{0}$ in Region IIb,

$$
\left(h_{-}-\left(r-r_{B}\right) \tan \alpha\right) \frac{\partial v_{0}}{\partial t}+\left(v_{0}-v_{B}\right)(\cos \alpha)^{-1 / 2}=\frac{K(t)}{r} .
$$

The quantity $2 \pi K(t)$ is the circulation of an irrotational vortex and its evaluation is non-trivial. Even were its value known, simultaneous solution of equations (2.22) and (2.28) is also problematic. For example, demanding continuity of $v_{0}$ at $r=r_{B}$, a straightforward assumption, leads to a problem since volumetric flow rates in the horizontal- and conical-boundary Ekman layers are given respectively by

$$
\begin{gathered}
F_{r}=-\frac{1}{2} E^{1 / 2}\left(v-v_{B}\right), \\
F_{\text {slope }}=-\frac{1}{2} E^{1 / 2}\left(v-v_{B}\right)(\cos \alpha)^{-1 / 2},
\end{gathered}
$$

so that the flow rate is not continuous at $r_{B}$, where the layers meet. Hence there must be a source/sink of fluid at $r_{B}$. The treatment of such a region is well established. (See, for example, Moore \& Saffman 1969b.) Nonetheless, the details are complicated, involving the Stewartson layers that straddle $r=r_{B}$. The analysis is presented in Appendix A, where we evaluate $K(t)$, and determine that the Laplace transform of the geostrophic velocity at $r=r_{B+}$ is given by

$$
\mathscr{L}\left\{v_{0 b}\right\}=\frac{r_{B}}{s}-\frac{r_{B}}{p^{2}} \frac{1+p q h_{-}}{(\cos \alpha)^{-1 / 2}+p q h_{-}},
$$

where

$$
p \equiv\left(s+h_{-}^{-1}\right)^{1 / 2}, \quad q \equiv\left(s+\left(h_{-}(\cos \alpha)^{1 / 2}\right)^{-1}\right)^{1 / 2} .
$$

Substitution of this result into the Laplace transform of (2.28) evaluated at $r=r_{B}$ determines the transform of $K(t)$ to be

$$
\mathscr{L}\{K(t)\} r_{B}^{-1}=\mathscr{L}\left\{v_{0 b}\right\} q^{2} h_{-}-r_{B}(s \sqrt{\cos \alpha})^{-1},
$$

from which we obtain the transformed version of (2.28),

$$
\left(s H_{-}+(\cos \alpha)^{-1 / 2}\right) \mathscr{L}\left\{v_{0}\right\}=\frac{r_{B} q^{2} h_{-}}{r} \mathscr{L}\left\{v_{0 b}\right\}+\frac{r^{2}-r_{B}^{2}}{r s(\cos \alpha)^{1 / 2}},
$$

with $H_{-}(r) \equiv h_{-}-\left(r-r_{B}\right) \tan \alpha$. We note here that $H_{-}(r)$ is clearly the depth of the fluid column at the radial location $r$. For brevity of notation we will use $H_{-}$in the formulae that follow, rather than showing the radial dependence explicitly.

Substitution of (2.31) into this expression and inversion of the Laplace transform 
leads to

$$
v_{0}(r, t)=r\left(1-\exp \left(-\mu t / H_{-}\right)\right)+\frac{\hat{h} r_{B}^{2}}{r} V_{0}(r, t)
$$

with

$$
\begin{aligned}
V_{0}(r, t)= & \exp \left(-\mu t / H_{-}\right)-\frac{\mu-1}{\mu C} \exp \left(-t / h_{-}\right)-\frac{D}{C} \frac{\mu^{1 / 2}-\mu(C D)^{1 / 2}}{\mu^{1 / 2}-(C D)^{1 / 2}} \exp \left(-\mu t / H_{-}\right) \\
& +2(\mu-1) \frac{3 \mu^{2}-2 \mu+1-(\mu-1) Q}{\mu Q(2 \mu \hat{h}-\mu-1-Q)} \exp \left(-(\mu+1+Q) t /\left(2 h_{-}\right)\right)+I(t)
\end{aligned}
$$

where $\mu \equiv(\cos \alpha)^{-1 / 2}, \hat{h} \equiv h_{-} / H_{-}, C \equiv \mu \hat{h}-1, D \equiv \hat{h}-1$, and $Q \equiv\left(5 \mu^{2}-2 \mu+1\right)^{1 / 2}$. In this expression, the function $I(t)$ comes from an integral around branch cuts in the $s$-plane, and is given by

$$
I(t) \equiv \frac{\mu-1}{\pi} \int_{1}^{\mu} \frac{(\mu-\phi)^{3 / 2}}{(\phi-1)^{1 / 2}} \frac{H_{-}}{h_{-}}\left(\mu^{2}+(\mu-\phi)(\phi-1)\right)^{-1} \exp \left(-\phi t / h_{-}\right) \mathrm{d} \phi .
$$

As we shall see in detail in $\S 2.4$, there is no Ekman layer beneath the interface at $z=0$. As noted already, neither is there an Ekman layer on the underside of the free surface. Hence, if we let $h_{+} \rightarrow 0$, then the results in this section also describe spin-up of a homogeneous fluid in a container of this shape. Furthermore, in the limit of $r_{B} \rightarrow 0$, the results of this section are in agreement with those presented by Greenspan \& Howard (1963).

\subsection{Spin-up in the cone/cylinder for $z_{c}>0$ : interface intersects the slope}

Figure 1 indicates the geometrical configuration in this situation. There are now four distinct regions: Ia,b and IIa,b. We deal with Regions I and II in detail below.

Region II: $z<0$. The analysis of the preceding section remains largely unchanged for the region $z<0$ since there are unsteady $E^{1 / 4}$ layers straddling $r=r_{B}$ as before. Thus, (2.23) describes the time history in Region IIa, where $r<r_{B}$, and solution (2.35) remains valid in Region IIb. However, the motion in Region I is somewhat different as will be discussed below.

Region I: $z>0$. There are no Ekman layers bounding Region Ia, and so the evolution is described by equation (2.25), as in the case explored in the preceding section. Therefore, on this time scale,

$$
v_{0}=0 \quad \text { in Region Ia: } z>0, r<a,
$$

where $a$ is the radial location where the interface intersects the sloping boundary. In Region Ib, the equation of motion is virtually identical to (2.28) except in $z>0$; it is

$$
\left(h_{+}-(r-a) \tan \alpha\right) \frac{\partial v_{0}}{\partial t}+\left(v_{0}-v_{B}\right)(\cos \alpha)^{-1 / 2}=\frac{K(t)}{r},
$$

where the circulation $2 \pi K(t)$ must once again be determined by considering the shear-layer structure at $r=a$. Evaluation of (2.39) at $r=a$ determines $K$ in terms of $\mathscr{L}\left\{v_{0 b}\right\}$, namely

$$
\left(s h_{+}+(\cos \alpha)^{-1 / 2}\right) \mathscr{L}\left\{v_{0 b}\right\}-a s^{-1}(\cos \alpha)^{-1 / 2}=\mathscr{L}\{K\} a^{-1} .
$$


Substitution for $K$ into (2.39) gives the transform in terms of $\mathscr{L}\left\{v_{0 b}\right\}$,

$$
\left(s H_{+}+(\cos \alpha)^{-1 / 2}\right) \mathscr{L}\left\{v_{0}\right\}=s^{-1}(\cos \alpha)^{-1 / 2}\left(r-\frac{a^{2}}{r}\right)+\frac{a}{r} h_{+}\left(q^{\prime}\right)^{2} \mathscr{L}\left\{v_{0 b}\right\},
$$

where

$$
H_{+} \equiv h_{+}-(r-a) \tan \alpha .
$$

The transform $\mathscr{L}\left\{v_{0 b}\right\}$ is discussed in detail in Appendix A, and is indeed complicated. The reason is that the shear layer in $r=a$ - has a width that grows in time, so, in fact, rather than as stated in (2.38), the swirl speed in Region Ia is small, in $E$, but not zero. It turns out that even small values of $v_{0 a}$ makes a difference in the form of $\mathscr{L}\left\{v_{0 b}\right\}$. Again, the details can be found Appendix A. The small- $E$ approximations lead to formula (A 20) for $\mathscr{L}\left\{v_{0 b}\right\}$, which may then be inverted in the usual way, leading to

$$
v_{0}(r, t)=r\left(1-\exp \left(-\mu t / H_{+}\right)\right)+\frac{a^{2}}{r} \tilde{V}_{0}(r, t),
$$

and that portion of $\tilde{V}_{0}$ on the spin-up time scale is given by

$$
\begin{aligned}
\tilde{V}_{0}^{(1)}(r, t)=\exp \left(-\mu t / H_{+}\right)-(1-\bar{h})\left\{1-[\bar{h}(\bar{h}-1)]^{1 / 2}\right\}^{-1} \\
\times \exp \left(-\mu t / H_{+}\right)-\frac{2 \mu(\mu-\Psi)}{(\mu-\Psi / \bar{h}) \sqrt{5} \Psi} \exp \left(-\Psi t / h_{+}\right)-J(t),
\end{aligned}
$$

where $\Psi=(1+\sqrt{5}) \mu / 2, \bar{h}=h_{+} / H_{+}$and $\mu=(\cos \alpha)^{-1 / 2}$ as before. The part of the transient due to an integral around branch points is given by

$$
J(t) \equiv \frac{1}{\pi} \int_{0}^{1}\left(\frac{(1-\phi)^{3}}{\phi}\right)^{1 / 2}\left(\left(1+\phi-\phi^{2}\right)(1-\phi / \bar{h})\right)^{-1} \exp \left(-\mu \phi t / h_{+}\right) \mathrm{d} \phi .
$$

However, this inversion does not capture the longer-time motion, again as discussed in Appendix A. In fact, with a small- $E$ approximation, other than those made to derive the 1/4- and 1/3-layer structures in the first place, we find that there is an origin pole in $\mathscr{L}\left\{v_{0 b}\right\}$, leading to an additional contribution to $\tilde{V}_{0}$. Solution (2.43) is therefore completed by including (2.44) and

$$
\tilde{V}_{0}(r, t)=\tilde{V}_{0}^{(1)}(r, t)+\tilde{V}_{0}^{(2)}(r, t),
$$

where

$$
\tilde{V}_{0}^{(2)}(r, t)=-\sqrt{\frac{\mu}{h_{+}}} \frac{E^{1 / 4}}{a} .
$$

\subsection{The time-dependent motion in the interfacial layer}

The general equations of motion for the interior of the fluid, away from horizontal and vertical viscous layers involve a shorter intrinsic time scale, namely the time scale of internal/inertial waves. To explore that, we introduce that short time scale as $\bar{t}=t E^{-1 / 2}$. Away from any high-gradient regions where viscous terms or diffusivity are important, equations (2.9)-(2.11) are easily reduced to a single equation for the vertical velocity,

$$
\frac{\partial^{2}}{\partial \bar{t}^{2}} \nabla^{2} w+4 \frac{\partial^{2} w}{\partial z^{2}}+\frac{S}{\delta^{2}} \exp \left(-z^{2} / \delta^{2}\right) \frac{1}{r} \frac{\partial}{\partial r}\left(r \frac{\partial w}{\partial r}\right)=0
$$


and the swirl velocity is related to $w$ by

$$
\frac{\partial}{\partial \bar{t}}\left(\frac{1}{r} \frac{\partial r v}{\partial r}\right)=2 \frac{\partial w}{\partial z} .
$$

Letting $\delta \rightarrow 0$ in these equations, with $z$ not small leads to equations that govern the propagation of inertial waves, which are associated with the initial stages of the spin-up in a way described by Greenspan \& Howard (1963). That wave propagation is of only second-order importance to the spin-up process, and is not further discussed here.

However, within the interfacial layer, the motion is much more complex. Putting $z=\delta \zeta$, the first term of an asymptotic expansion in $\delta$ for the velocity and density in that region gives, from (2.48), the equations for internal-gravity/internal waves,

$$
\begin{gathered}
\left(\frac{\partial^{2}}{\partial \bar{t}^{2}}+4\right) \frac{\partial^{2} w}{\partial \zeta^{2}}+S \exp \left(-\zeta^{2}\right) \frac{1}{r}\left(r \frac{\partial w}{\partial r}\right)=0, \\
\delta \frac{\partial}{\partial \bar{t}}\left(\frac{1}{r} \frac{\partial r v}{\partial r}\right)=2 \frac{\partial w}{\partial \zeta} .
\end{gathered}
$$

This interfacial layer is thicker than the Ekman-layer thickness provided that

$$
\delta \gg E^{1 / 2},
$$

a restriction already noted in (2.8). This restriction means that viscous diffusion is absent from the equations describing the motion in this $\delta$-layer. Since solutions of (2.50) are continuous, there is no need for a thinner Ekman layer for smoothing of the flow quantities. Should $\delta=O\left(E^{1 / 2}\right)$, however, the dynamics of this layer must include the effects of viscous diffusion, leading to a much more complicated description than that provided by (2.50). For values of $\delta$ much smaller than $E^{1 / 2}$, there are indeed Ekman layers that sandwich a much thinner $\delta$-layer. In the experiments to be described in $\S 4$, the Ekman number is $E \approx 4.78 \times 10^{-5}$, for which the $E^{1 / 2}$-scale is smaller than the width of the transition zone, for which $\delta \approx 0.075$.

For the theoretical model presented here, we are assuming that deflection of the free surface and transition zone during the readjustment phase has a negligible effect at leading order over the $E^{-1 / 2} \Omega^{-1}$ timescale. Comments regarding the applicability of this assumption to the experimental component of this work are made in $\S 4$.

Vertical-velocity boundary condition for the outer flow

Note that, from (2.51), the vertical velocity in this region is $O(\delta)$, and on the spin-up time scale of $\S 2.4 .2$, it is even smaller $-O\left(\delta E^{1 / 2}\right)$. Therefore, to match to the regions away from this layer, on a spin-up time scale $w=o\left(E^{1 / 2}\right)$, and hence this region acts as a solid boundary to the geostrophic flow above and below, requiring that the $O\left(E^{1 / 2}\right)$ vertical velocity component vanish at $z=0$, leading to the boundary condition already utilized as (2.17).

\subsubsection{Motion for short times}

Since it turns out that where this layer intercepts a sloping or vertical wall, the radial velocity component, $u$, must be zero (see below), we introduce the following representation for $w$ :

$$
w=\sum_{n=1}^{\infty} A_{n}(\zeta, \bar{t}) J_{0}\left(\frac{j_{n} r}{a}\right),
$$


where $j_{n}$ is the $n$th zero of the Bessel function $J_{1}$, and hence satisfies the boundary condition on $u$. Substitution of (2.53) into (2.50)-(2.51), followed by a Laplace transform in time, leads to

$$
\frac{\partial^{2}}{\partial \zeta^{2}} \overline{\mathscr{L}}\left\{A_{n}\right\}=\frac{S \exp \left(-\zeta^{2}\right) j_{n}^{2}}{a^{2}\left(\bar{s}^{2}+4\right)} \overline{\mathscr{L}}\left\{A_{n}\right\},
$$

where the overbar on $\mathscr{L}$ indicates a Laplace transform variable $\bar{s}$, related to the (short) time variable, $\bar{t}$.

Considerable analysis has been done regarding the nature of the solution to (2.54). We have not included the details here, since they have little effect on the global spin-up problem, but we give a brief summary below.

Matching of solutions to (2.54), to either Region Ia or Ib above, and to either Region IIa or IIb below, introduces various Laplace-domain singularities, in addition to those inherent in the solutions to (2.54) itself. Without giving the details, we note that there are a number of poles of $\overline{\mathscr{L}}\left\{A_{n}\right\}$ a distance $E^{1 / 2}$ from the origin, obviously related to behaviour on the spin-up time scale. There are also two sets of branch points on the same scale, leading to features that decay algebraically in time. More important to the short-time behaviour, there are branch points of $\overline{\mathscr{L}}\left\{A_{n}\right\}$ that are located at $\bar{s}= \pm 2 \mathrm{i}$. These branch points contribute inertial oscillations with algebraically decaying amplitudes.

\subsubsection{Motion on the spin-up time scale}

On the spin-up time scale, we may neglect the time derivatives in (2.50), in which case the equation takes the form

$$
\frac{\partial}{\partial r}\left(\frac{1}{r} \frac{\partial r v_{0}}{\partial r}\right)+\frac{S}{4} \frac{\partial}{\partial \zeta}\left(\exp \left(\zeta^{2}\right) \frac{\partial v_{0}}{\partial \zeta}\right)=0 .
$$

(It is more convenient to work directly with the swirl velocity here.) In spite of the apparent time-invariance on this scale, matching conditions at large $|\zeta|$ contain unsteadiness on the spin-up time scale, as reflected in the presence of the $\bar{s} S=O\left(E^{1 / 2}\right)$ poles noted in the previous section.

In particular, for the case of spin-up in a cylinder, as discussed in $\S 2.2$, the matching conditions on $v_{0}$ are given by (2.23) and (2.25), and are

$$
\begin{gathered}
v_{0} \rightarrow 0 \text { for } \zeta \rightarrow \infty, \\
v_{0} \rightarrow r\left(1-\exp \left(-t / h_{-}\right)\right) \text {for } \zeta \rightarrow-\infty .
\end{gathered}
$$

A solution of (2.55) for this case is

$$
v_{0}=\frac{1}{2} r\left[1-\exp \left(-t / h_{-}\right)\right] \operatorname{erfc}(\zeta),
$$

but it is valid only in the central portion of the layer, since it fails to satisfy the condition $u=0$ at the container wall.

For the case $z_{c}<0$ (for which the density interface intersects the vertical wall), the solution is somewhat more complicated, but the large-time behaviour is equivalent to that found in the cylinder geometry (2.58), and is

$$
v_{0} \rightarrow \frac{1}{2} \operatorname{rerfc}(\zeta) \text { as } t \rightarrow \infty,
$$

which is valid all the way to the wall in this limit.

However, for the case of $z_{c}>0$ as discussed at the beginning of this subsection, even the long-time behaviour is complicated. Nevertheless, the numerical solution to (2.55) is easy to obtain although we do not present any such results here. 


\subsubsection{On the radial-velocity boundary condition}

The question of what boundary conditions are to be imposed on the solution of (2.55) needs to be addressed. Since there is an obvious symmetry condition at $r=0$, the question is about the condition to be applied at the wall, $r=r_{w}(=1$ for $z_{c}<0$ and $R$ for $z_{c}>0$ ). Above this layer, the swirl velocity matches to a diffusively controlled flow in which there is a boundary layer at $r=r_{w-}$ that has width $\left(E^{1 / 2} t\right)^{1 / 2}$ on this time scale, and the interior is not spun up. Hence $v$ matches to zero and the inflow into the layer is slight, so $u=0$ at $r=r_{w}$ at large positive $\zeta$. However, in Region II, for all of the cases studied, there is an $O\left(E^{1 / 2}\right)$ inflow into the sidewall 'quarter layer'. Thus, for large negative $\zeta$, it appears that $u$ is not zero at $r=r_{w}$. Resolution of what condition is to be imposed requires a careful examination of the $E^{1 / 4}$ layer on $r=r_{w-}$, which is complicated by the exponential behaviour of $N^{2}$. Such an examination reveals that the usual Stewartson 'quarter layer', with its concomitant $O\left(E^{1 / 2}\right)$ inflow, persists along the side of this thicker interfacial layer only for negative $\zeta$ values larger than $O\left([-\log E]^{1 / 2}\right)$. On the other hand, for $O(1)$ values of $\xi$, the large stratification attenuates the vertical motion, so that alongside the $O(1)$ interfacial layer the radial velocity is $O\left(\delta E^{1 / 4}\right)$, which is sufficiently small for $u=0$ to be the appropriate condition. The transition between these regions occurs in a layer of width $E^{1 / 4}$, but with a $z$-thickness of $O\left(\delta /(-\log E)^{1 / 2}\right)$, at a $z$-distance of $O\left(\delta(-\log E)^{1 / 2}\right)$. Further investigation indicates that the net result is a very weak singularity that arises near the edge of the interfacial layer, and at $r=r_{w}$, but its global effect on the spin-up is minimal, and hence it is appropriate to put $u=0$ for all $r=r_{w}$.

\section{Summary of theoretical predictions}

On considering the spin-up of Region II, as given by (2.35), and the different spin-up mechanisms in Region I for the two cases under study, a few points may be noted.

When $z_{c}<0$, Region I does not spin up at all on the 'fast' time scale, so that

$$
v \equiv 0 \quad \text { as } \quad t \rightarrow \infty \quad \text { in Region } \mathrm{I}, z_{c}<0 .
$$

Below the interface, since for (2.37)

$$
I(t) \sim\left(\frac{h_{-}}{\pi t}\right)^{1 / 2} \frac{(\mu-1)^{5 / 2}}{\mu^{2}} \frac{\exp \left(-t / h_{-}\right)}{\mu h_{-} / H_{-}-1} \text { for } t \rightarrow \infty,
$$

equation (2.35) indicates that

$$
v_{0} \rightarrow r+\mathrm{EST} \text { for } t \rightarrow \infty \text { in Region } \mathrm{IIb}, z_{c}<0,
$$

and, from (2.23),

$$
v_{0} \rightarrow r+\mathrm{EST} \text { for } t \rightarrow \infty \text { in Region IIa, } z_{c}<0 .
$$

In these expressions, 'EST' stands for exponentially small terms. Hence, all of Region II is spun up on this time scale, and all of Region I is still rotating at the initial angular frequency. In this sense there is no qualitative difference between this case and that of a circular cylinder, as discussed in $\S 2.1$.

Now, we turn to the results for $z_{c}>0$. As noted in the preceding section, all of Region II responds to the forcing in a way that is unchanged from the $z_{c}<0$ case, so

$$
v_{0} \rightarrow r+\mathrm{EST} \text { for } t \rightarrow \infty \text { in Region II, } z_{c}>0 \text {. }
$$




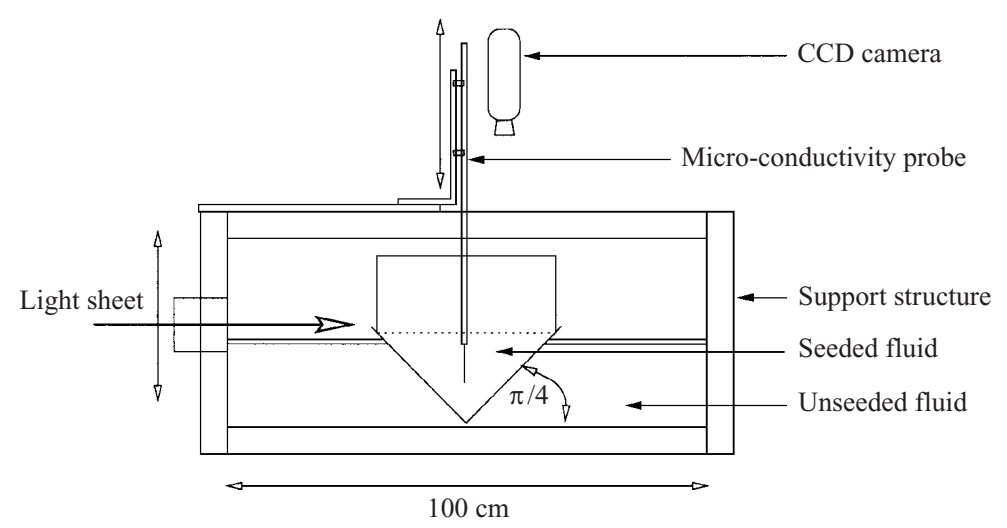

FIGURE 2. Experimental apparatus. The structure shown is mounted on a levelled, computer-controlled turntable with the CCD camera fixed in the rotating frame of reference.

However, examination of the Region Ib solution reveals some unusual features. First, notice that (2.45) gives

$$
J(t) \sim \sqrt{\frac{h_{+}}{\pi \mu t}} \text { for } t \rightarrow \infty
$$

and therefore (2.43) indicates that

$$
v_{0} \sim r-\frac{a^{2}}{r} \sqrt{\frac{\mu}{h_{+}}} \frac{E^{1 / 4}}{a}-\frac{a^{2}}{r} \sqrt{\frac{h_{+}}{\pi \mu t}}+\text { EST for } t \rightarrow \infty \quad \text { in Region } \mathrm{Ib}, z_{c}>0 .
$$

So, there is a slow algebraic decay toward a partially spun-up state in this zone. In Region Ia, no readjustment will be achieved on this time scale

$$
v \equiv 0 \text { for } t \rightarrow \infty \text { in Region Ia, } z_{c}>0 .
$$

In summary, regardless of the location of the interface (above or intersecting the slope) the lower layer fully spins up on this time scale, but there is a difference in the region above the interface: in Region I, any zone of fluid that lies over the sloping wall and not over the interface does partially spin up, but slowly and algebraically; however, the central region above the interface remains unaffected by the forcing since the readjustment mechanism in this region acts over a diffusive time scale.

\section{Laboratory experiments}

\section{The apparatus}

A set of laboratory experiments has been performed to test the conclusions of the previously detailed linear spin-up analysis. The laboratory apparatus is shown schematically in figure 2, and a representative set of experimental parameters is given in table 1.

We utilize a composite geometry, which is constructed from a high-precision acrylic conical shell acting as the base of a similarly fabricated circular cylinder. Both the cylinder and the cone are transparent, allowing illumination of tracer particles (for particle tracking purposes) by a horizontal light sheet of typical depth $6 \times 10^{-3} \mathrm{~m}$. The inner radius of the cylindrical shell is $0.1825 \mathrm{~m}$ and the lower conical surface is at an angle $\pi / 4$ to the horizontal. The lower rim of the cylinder is machined to approximate 


\begin{tabular}{lcccccc}
\hline Expt. & $\Omega\left(\mathrm{rad} \mathrm{s}^{-1}\right)$ & $R o$ & $E$ & $h_{-}=a$ & $h_{+}$ & $\Delta \rho\left(\mathrm{kg} \mathrm{m}^{-3}\right)$ \\
1 & $\pi / 5$ & $1 / 6$ & $4.78 \times 10^{-5}$ & 1.48 & 0 & 0 \\
2 & $7 \pi / 30$ & $1 / 7$ & $5.68 \times 10^{-5}$ & 1.00 & 0.50 & 8 \\
3 & $\pi / 5$ & $1 / 4$ & $4.78 \times 10^{-5}$ & 0.6 & 0.93 & 17 \\
4 & $\pi / 5$ & $1 / 4$ & $4.78 \times 10^{-5}$ & 0.275 & 1.26 & 13 \\
5 & $\pi / 5$ & $1 / 6$ & $4.78 \times 10^{-5}$ & 1.23 & 0.33 & 10 \\
6 & $\pi / 5$ & $-1 / 3$ & $4.78 \times 10^{-5}$ & 0.58 & 1.0 & 10
\end{tabular}

TABLE 1. A representative sub-set of experimental parameters for which results are presented.

the angle of the conical wall, and the two pieces are joined with a small quantity of a silicon sealant. This composite container is placed above the axis of rotation of the turntable and is affixed to a support plate in order to maintain its position. A larger surrounding structure allows an unseeded quantity of fluid to be introduced around the lower portions of the container. This minimizes the forces exerted on the support plate and reduces the optical difficulties of maintaining a horizontal light sheet through the sloping lower container wall.

In terms of the parameters defined in the previous theoretical work, this configuration corresponds to $R=0.1825 \mathrm{~m}$ and $r_{B} \ll 1$.

The structure shown in figure 2 is mounted on a levelled turntable, the rotational speed of which can be controlled accurately through a remote electrical control system. The rotation changes are initiated by specifying a final rotation rate and a constant acceleration level in the input to the motor controller. For small changes in the rotation rate of the turntable, the initial and final angular frequency of the container are accurate to $\pm 0.002 \mathrm{rad} / \mathrm{s}^{-1}$.

Filling of the cylinder/cone is performed with the container at rest; a volume of fresh water is introduced into the tank, followed by a prescribed quantity of a salt solution. The more dense lower layer (the saline water) is fed slowly under gravity to the apex region of the conical base via a small filling-tube leading from an external reservoir. The tube is then removed and the system is allowed to spin up to a state of rigid body rotation. Spin-up of the fluid/container system is achieved by a slow acceleration of the turntable towards a fixed final rotation rate; this avoids excessive interfacial mixing.

\section{Data acquisition}

Following a change in the rotation rate of the container, quantitative data are obtained for the transient response of the system through a combination of particle tracking techniques and density measurements.

The azimuthal flow field at a specified depth in the flow can be visualized by the introduction of a sufficient number of neutrally buoyant particles and their illumination by a horizontal light sheet. However, since the linear analysis presented in the previous sections suggests that the interior flow evolution should be independent of the vertical coordinate (in each layer), most data relevant to the upper layer evolution are obtained from particles distributed over the free surface. The illuminated particle motion is recorded onto video cassette for later processing with particle tracking software, as discussed by Dalziel (1992).

A calibrated aspirating conductivity probe is used to measure the density of the working fluid at a specified location. The probe can be deployed in one of two modes during an experiment, either attached to a vertical traverse that enables density 
profiles to be obtained over a depth range, or stationary to provide time-series data. A solid metal electrode is immersed unobtrusively elsewhere in the fluid to complete the electrical conductivity circuit.

\section{Parameter regimes}

Experiments are performed with non-dimensional parameters in the range

$$
\begin{array}{ll}
|R o| \in[1 / 7,1 / 3] & B u \in[0,6], \\
r_{B} \ll 1, & h=\left(h_{+}+h_{-}\right) \in[1.48,1.59], \\
\sigma=700, & E \approx 5 \times 10^{-5}, \\
a \in[0.25,0.75], & h_{-} \in[0.3, h],
\end{array}
$$

together with

$$
\begin{array}{ll}
\Omega \in[\pi / 5,7 \pi / 30] \mathrm{rad} \mathrm{s}^{-1}, & R=0.1825 \mathrm{~m}, \\
v=10^{-6} \mathrm{~m}^{2} \mathrm{~s}^{-1}, & N^{2} \in[3,30] \mathrm{s}^{-2} .
\end{array}
$$

Note that the Froude number based on the radius of the cylinder and maximum fluid depth is

$$
F=4 \Omega^{2} R^{2} / g h^{*}<0.02,
$$

for which a maximum steady deviation of the parabolic free surface during rigid body rotation is considerably smaller than the total depth.

The laboratory configuration is such that $r_{B} \ll 1$, and therefore we do not present any quantitative comparison with predictions of the evolution in Region IIa. The fluid below the interface is simply referred to as Region II in the subsequent discussion.

\section{The interfacial region}

Laboratory results confirm that, for sufficiently small $R o$ and $F$, the large-scale response of the two layers (on a $E^{-1 / 2} \Omega^{-1}$ time scale) is independent of the density difference (for $\Delta \rho \in[4,35] \mathrm{kg} \mathrm{m}^{-3}$ ). The effect of the density interface is to effectively isolate the regions of fluid above and below it, leading to largely independent spin-up responses. We therefore concentrate on making quantitative comparisons of the linear theory with particle tracking results obtained throughout the flow, and discuss the evolution of the density interface in somewhat less detail.

The characteristic spin-up times are different in each of the two main layers, therefore a force imbalance during the transient response will lead to a 'doming' of the initially parabolic interface. This displacement of the interface is very small relative to the depth of the individual fluid layers. Small-scale interfacial displacement can be measured via a density probe at a fixed location in the interfacial layer. The unsteady displacement of the isopycnals can be measured via the shift in the density measured at a fixed point when the probe is centred in the high- $N$ region.

A simple method of presenting the density measurements is to assume that the density is a linear function of the vertical coordinate near to the mid-plane of the interface. That is, for a measured density of $\rho_{m}(t)$ (relative to an initial value of $\rho_{0}$ ), we can define a dimensionless interfacial shift at the radial location of the probe to be

$$
\Delta=\left|\frac{g \rho_{m}(t)}{\rho_{0} N_{\max }^{2} R}\right|,
$$

where $N_{\max }$ is the peak value of the buoyancy frequency. The quantity $\Delta$ is an approximate measure of the interfacial shift relative to the natural length scale, which in this case is taken to be the cylinder radius. Such measurements show a peak interfacial displacement of the order of just a few percent of the container radius $R$. The 


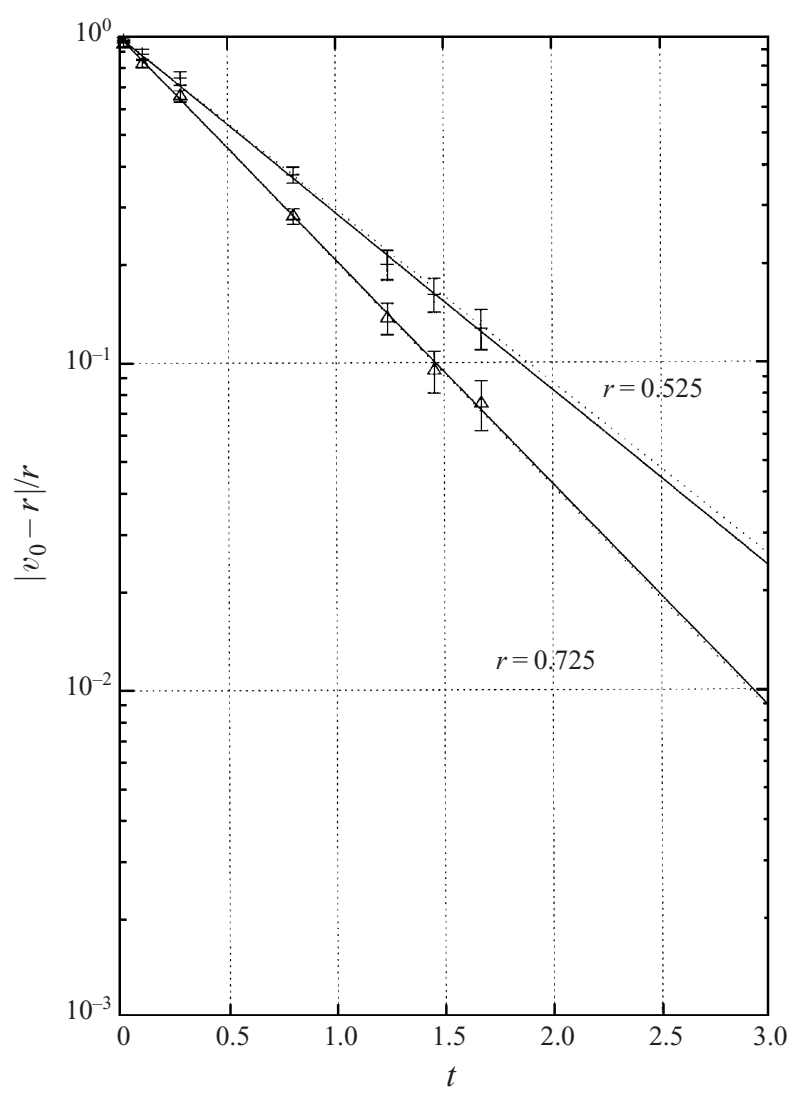

FIGURE 3. Decay of the local normalized angular frequency at two radial locations in Region II for experiment 1 (see table 1). The error bars on the data points indicate the standard deviation of the mean and the solid lines are predictions based on the linear theory. The dotted lines are generated by linear regression through the data.

linear character of the response can be verified by comparing data at $R o= \pm \epsilon$ for a sufficiently small value of $\epsilon$; the same response (to within experimental accuracy) can be obtained with only a sign change.

Obviously, the interfacial displacement must have some functional dependence on $R o$ and $B u$ as well as the other parameters associated with the container geometry. However, for sufficiently small $R o$ the displacement is small and is of little importance to the linear spin-up mechanisms on the $E^{-1 / 2} \Omega^{-1}$ time scale for $h_{-}, h_{+} \sim O(1)$. Therefore, we do not investigate the quantitative behaviour of the interfacial region in detail.

\section{Experimental results and comparisons}

\subsection{Region II: spin-up response below the density interface}

The unsteady response of Region II is predicted to be qualitatively independent of the location of the interface relative to the discontinuity in wall slope. In fact, the analysis is equally valid for the response of a contained volume of homogeneous fluid. A set of reference laboratory experiments was performed with a homogeneous fluid 


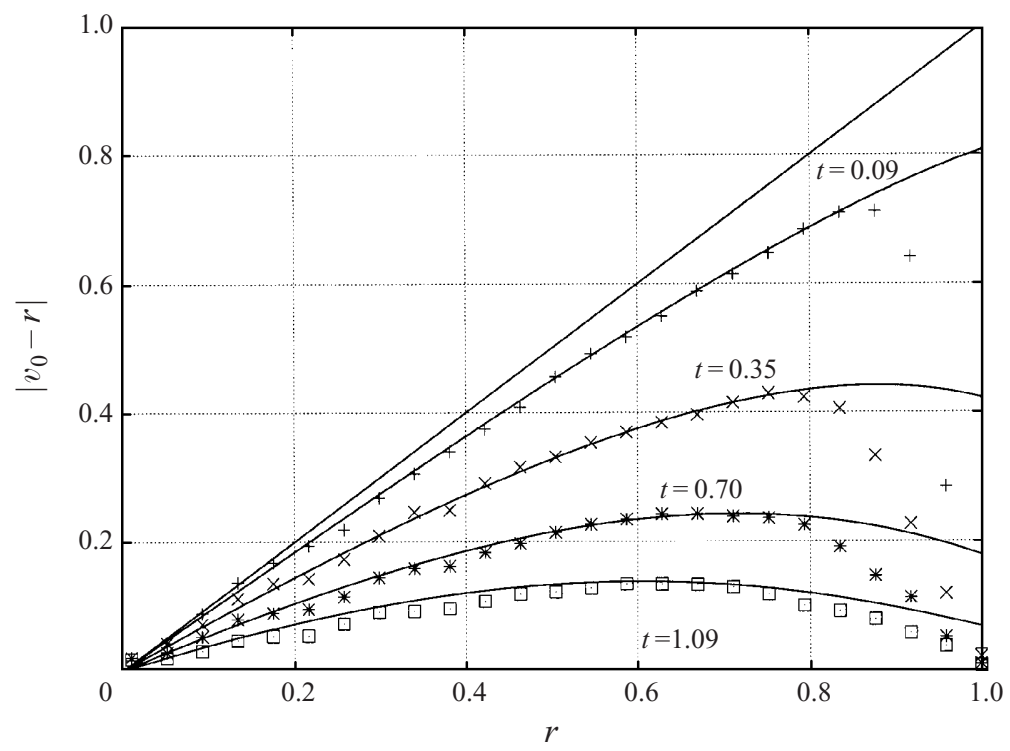

FIGURE 4. A comparison between laboratory results (symbols) and the inviscid interior solution (solid lines) for the azimuthal velocity in Region II for experiment 1 . Since the container is of variable depth, the azimuthal velocity profile is not simply proportional to $r$ during the transient response.

(no density interface) in the composite geometry. Particle tracking data were obtained from the free surface.

Figure 3 presents data for the evolution of the local, normalized angular frequency of the fluid at two radial locations. Since the camera views the motion relative to the final rotation rate of the container $\left(\Omega+\Delta \Omega s^{-1}\right)$, the quantity shown is

$$
\bar{\omega}=\left|\frac{v_{0}-r}{r}\right| .
$$

The figure presents results for $r \approx 0.525$ and $r \approx 0.725$, with error bars indicating the standard deviation of the mean. The data for each case are averaged over the radial intervals of $0.5 \leqslant r \leqslant 0.55$ and $0.7 \leqslant r \leqslant 0.75$, and the theoretical predictions are made with $r=0.525$ and $r=0.725$ respectively.

The linear spin-up analysis predicts (from $(2.25)$ with $r_{B} \ll 1$ ) that

$$
\left|\left(v_{0}-r\right) / r\right|=\exp \left(-\mu t / H_{-}\right)+O\left(r_{B}^{2}\right), \quad H_{-}=h_{-}-r,
$$

and it is this quantity that is represented by the solid lines in figure 3 .

A comparison of the predicted azimuthal velocity in Region II is presented in figure 4, which shows good agreement away from the viscous sidewall layer. The data of figure 4 are obtained by particle tracking at the free surface (for experiment 1 , see table 1) over a period of $1 \mathrm{~s}$, then azimuthally averaging and computing a segmented average over the radial coordinate.

As can be seen from figures 3 and 4 , the experimental method reproduces the classical linearized spin-up evolution. Similar results can be obtained for spin-up in a cylindrical container with a horizontal base, in which case the constant fluid depth leads to an interior response that is independent of the radial coordinate; see Greenspan \& Howard (1963).

In cases where a density interface exists, the response in Region II remains the same 


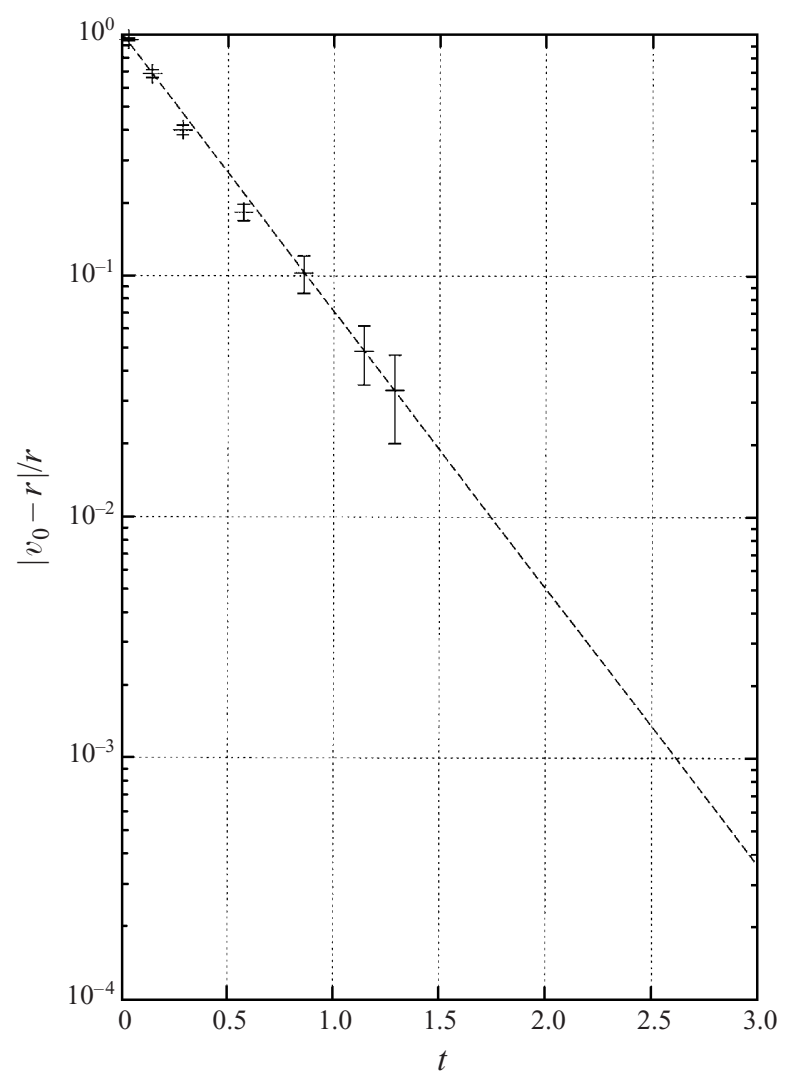

FIGURE 5. Decay of the local normalized angular frequency near $r=0.55$ in Region II for experiment 2. The error bars indicate the standard deviation of the mean and the dashed line is a prediction based on the linear theory.

in the theoretical treatment. Figure 5 shows a comparison between the theory and experiments for decay of the local normalized angular frequency in Region II during experiment 2 . The data are obtained from neutrally buoyant particles introduced into the lower layer, which are illuminated from the side via a horizontal light sheet and viewed from above. Particle tracking is performed over the interval $0.5 \leqslant r \leqslant 0.6$, and a theoretical prediction is given for $r=0.55$, as shown by the dashed line.

As may be anticipated, spin-up in Region II is achieved through a mechanism of secondary meridional circulation. The quantitative response is essentially that outlined by Greenspan \& Howard (1963) since the region is bounded below by an Ekman layer.

\subsection{Region I: spin-up response above the density interface}

An interface intersecting the vertical wall of the cylinder

For an interface intersecting the vertical upper wall of the container, the response in the lower layer is as outlined above. However, since the upper layer is not bounded by Ekman layers on solid boundaries, there is no dominant induced meridional circulation and the previous analysis predicts that readjustment occurs via viscous diffusion.

In figure 6 we present data obtained in experiment 5 for the variation of the 


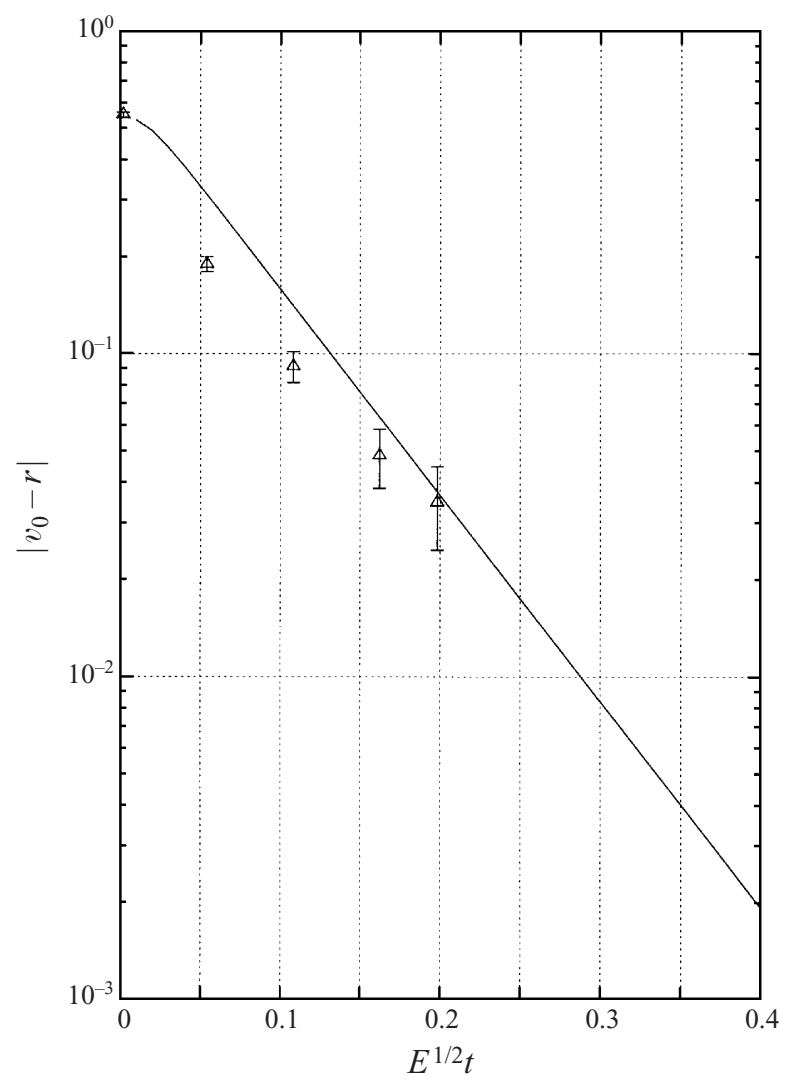

FIGURE 6. Decay of the azimuthal velocity in Region Ia for experiment 5 . The density interface intersects the vertical wall and the time scale is $t^{\prime}=t^{*} v / R^{2}=E^{1 / 2} t$. The solid line is a theoretical prediction and the data points are obtained from a representative laboratory experiment in the region $r \in[0.5,0.6]$.

azimuthal velocity near $r=0.55$. The time scale is that relevant for viscous diffusion $t^{\prime}=t^{*} v / R^{2}=E^{1 / 2} t$, and the density interface in this case is above the discontinuity in slope of the container.

The data cover a dimensional time scale of nearly two hours in this experiment and the solid line is the theoretical prediction based on summing the first ten terms in the series (2.18). As can be seen from the figure, the qualitative assumption of the linear analysis is verified, that is, Region Ia readjusts over a much longer time scale than a region bounded by an Ekman layer. Nevertheless there is a significant deviation between the predicted response and the laboratory data.

The discrepancy illustrated by figure 6 is typical of data obtained in a number of experiments and is attributable to the presence of some slight meridional circulation or nonlinear effect in the upper layer that remains sufficient to dominate the weak diffusive spin-up mechanism. Following the comments of a referee, we note that since there is no dominant Ekman recirculation in this region, the otherwise small freesurface/interface deflection may induce some recirculatory motion that will lead to a quantitative difference from the predictions of the theory. However, the readjustment time scale for this region is seen to be much slower (though not necessarily $E^{1 / 2}$ slower) than the other regions, which is the crucial assumption in the theoretical model for predictions over the $E^{-1 / 2} \Omega^{-1}$ time scale in the adjacent regions. 
An interface intersecting the sloping wall of the cone

When the density interface intersects the sloping base of the container, there exists a sub-region of $\mathrm{I}$ (denoted by $\mathrm{Ib}$ ) that is an annular region of fluid bounded by a free-standing shear layer, a Stewartson layer at the wall, a free surface and an Ekman layer.

The analysis of the previous sections predicts that the response within this region is of the form

$$
v_{0}-r=-r \exp \left(-\mu t / H_{+}\right)+\frac{a^{2}}{r} \tilde{V}_{0}(r, t),
$$

where $H_{+}=h_{+}-(r-a), \mu=(\cos \alpha)^{-1 / 2}$ and $\tilde{V}_{0}$ is given by (2.46).

There are some experimental difficulties in making quantitative observations of Region Ib over a significant time scale. A typical Ekman number for the laboratory experiments is $E \approx 5 \times 10^{-5}$, and the viscous layers bounding Region $\mathrm{Ib}$ are of dimensionless width $E^{1 / 4}$. Therefore, to separate the viscous layers at $r=a$ and $r=1$, one must make $a$ small. However, for $a \ll 1$ the partially spun-up state predicted by the theory will be very weak and difficult to observe in the laboratory.

Although the general transient behaviour is complicated to evaluate, there are simple expressions available for the small- and large-time behaviours that can be used for making quantitative comparisons. When the radius of the density interface is small in comparison to the radius of the cylinder, that is $a \ll 1$, we expect the initial response of the fluid in Region $\mathrm{Ib}$ to be

$$
\left|v_{0}-r\right|=r \exp \left(-\mu t / H_{+}\right)+O\left(a^{2}\right) .
$$

However, for sufficiently large $t$, terms of $O\left(a^{2}\right)$ are significant in the expression for the azimuthal velocity. Therefore, we expect that an algebraic approach to a partially spun-up state should eventually be observable. In this state the flow should approximate

$$
\left|v_{0}-r\right|=\frac{a^{2}}{r}\left(\sqrt{\frac{\mu}{h_{+}}} \frac{E^{1 / 4}}{a}-\sqrt{\frac{h_{+}}{\pi \mu t}}\right)+\text { EST. }
$$

Particle tracking experiments have been performed within Region $\mathrm{Ib}$, the results of which are shown in figures 7(a) and 7(b) for two different values of $a$. The figure shows the decay in measured azimuthal velocity over the non-dimensional homogeneous spin-up time scale $t=E^{1 / 2} \Omega t^{*}$. Each part presents an experimental time series formed from a running average of particle tracking data at a fixed radial location, together with the two theoretical predictions (5.4) and (5.5). As can be observed from the figure, there is good quantitative agreement concerning the deviation from an exponential decay towards a partially spun-up state on this time scale.

In figure 8 we show a comparison of experimentally determined profiles of angular frequency with the theoretically predicted values for Region Ib. Away from the shear layer straddling $r=a$ and the viscous layer at the cylindrical wall $(r=1)$ there is good agreement between the theory and laboratory data. We note that particle tracking data are unreliable when close to the axis of rotation due to the poor pixel resolution of the small-radius paths.

\subsection{The qualitative behaviour for $R o=O(1)$}

Experiments have been conducted in which the change in rotation is sufficiently large (relative to the background rotation) to introduce nonlinear effects. Typically, there is a limiting value of the Rossby number beyond which a distinct change in the evolution 

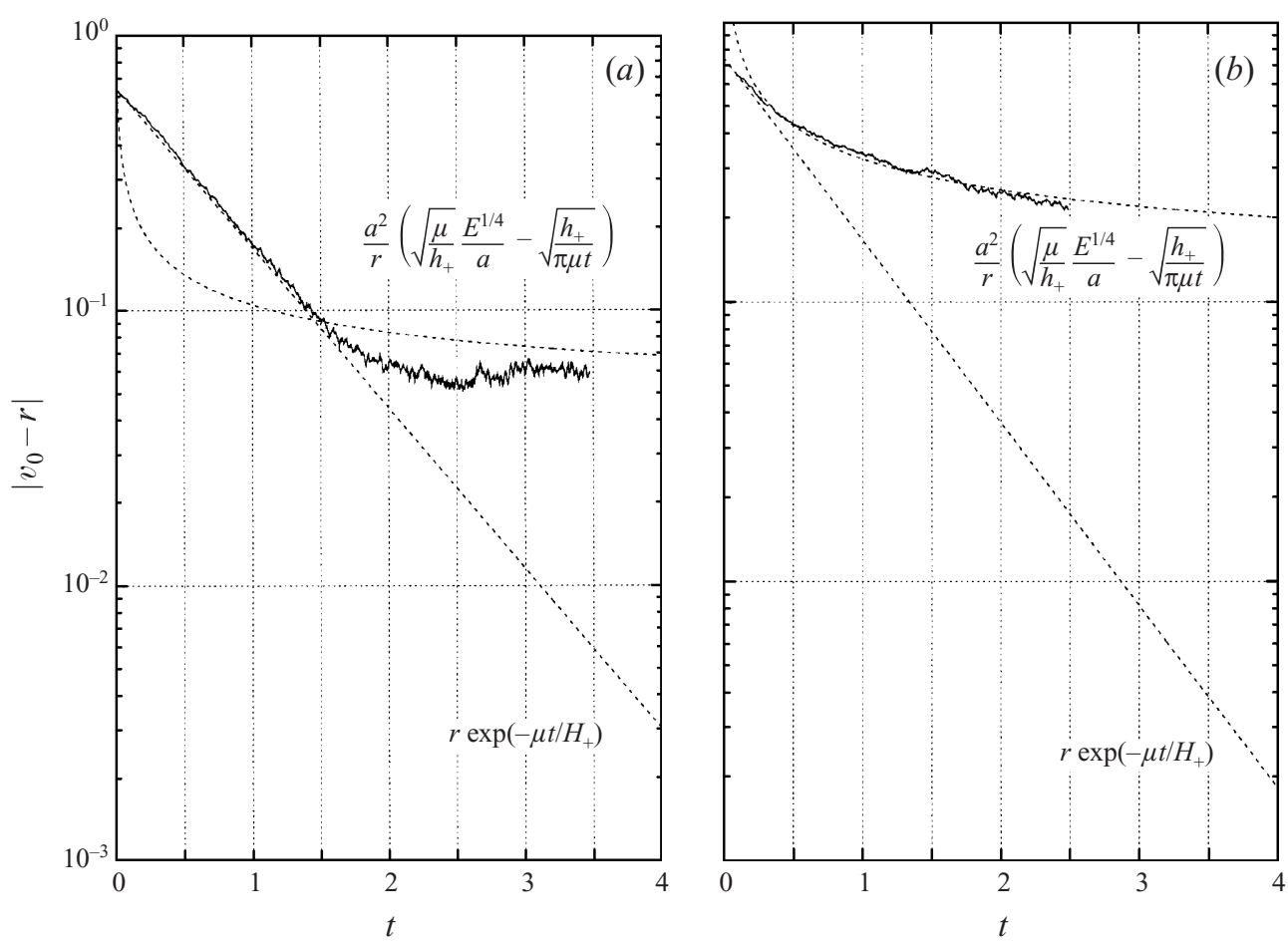

FIGURE 7. (a) Decay of the azimuthal velocity in region $\mathrm{Ib}$ for experiment 4 . The theoretically predicted partially spun-up state (for large time) and initial response are shown as dashed lines. The data were obtained at a mean radial location of $r=0.65$. (b) As $(a)$ but for experiment 3 with data obtained at $r=0.75$.

is obtained. For Rossby numbers below the limiting value the qualitative response remains as described previously, but with an increasing quantitative discrepancy between theory and experiment. (No comparisons have been made with improved estimates of Ekman transport at larger values of Ro.) A complete experimental investigation of the $R o=O(1)$ regime is beyond the scope of this paper; however we shall mention some generic features associated with such flows.

As the Rossby number is increased, density information gathered within the interface suggests that an oscillation of the interfacial displacement can be observed. These oscillations are more pronounced away from the axis of rotation and can be found for both positive and negative Rossby numbers.

In cases of nonlinear spin-down with the density interface intersecting the sloping wall, the transient response typically leads to a non-axisymmetric flow in the region near to the interface. On reducing the angular frequency of the container, the Ekman layer on the sloping wall in the upper layer leads to a downwards mass transport that impinges upon the interface. This leads to a roll-up of the fluid, which becomes unstable and undergoes a rapid breakdown, breaking the axisymmetry. The vertically displaced (denser) fluid then mixes rapidly and the flow can subsequently evolve through states with an azimuthal periodicity, although the final state is obviously fixed to be a rigid body rotation.

In figure 9, a sequence of frames obtained during nonlinear spin-down is shown (experiment 6). In this case the lower layer is dyed with fluoroscene and a horizontal 


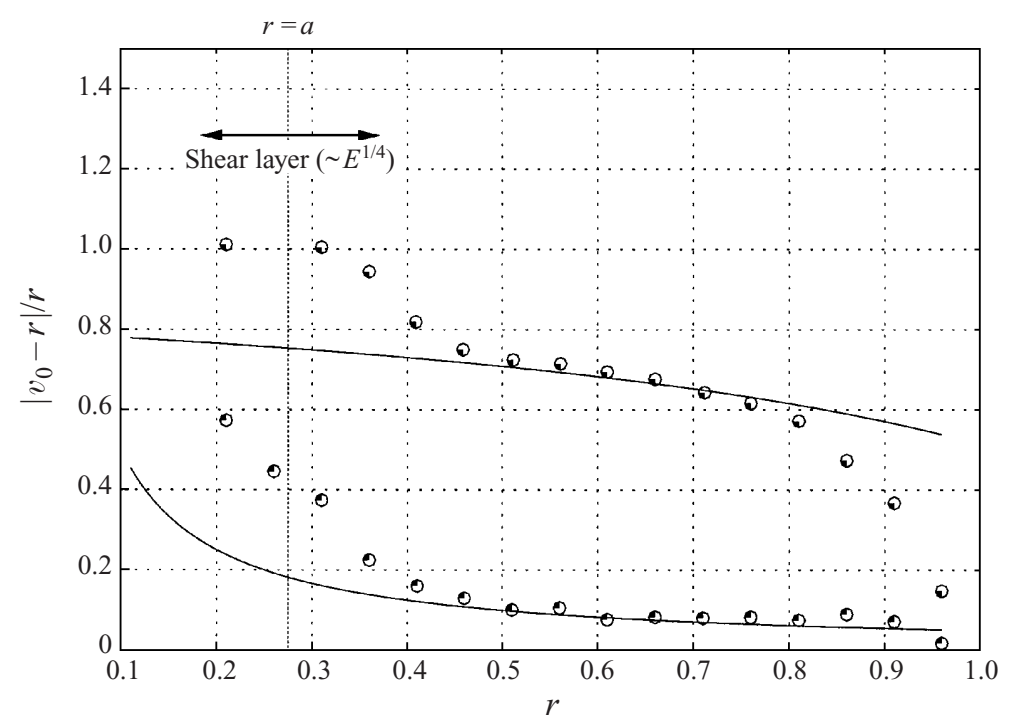

FIGURE 8. A comparison of profiles of local angular frequency $\left|\left(v_{0}-r\right) / r\right|$ for experiment 4 in Region I. The location of the shear layer separating Regions Ia and Ib is shown by the vertical line at $r=a$. Theoretical predictions of the angular frequency distribution in Region $\operatorname{Ib}(a \leqslant r \leqslant 1)$ are shown (solid lines), together with experimentally determined values (symbols). The two profiles are obtained at non-dimensional times $t=0.3,2.8\left(t=E \Omega t^{*}\right)$, and the theoretical predictions are made using the short- and large-time descriptions respectively, equations (5.4) and (5.5).

light sheet is positioned approximately $1 \mathrm{~cm}$ above the top of the dyed fluid (whilst the system is at rest and the dye interface is horizontal). The system is allowed to slowly spin up to an angular frequency of $\pi / 5 \mathrm{rad} \mathrm{s}^{-1}$ then the rotation rate is decreased to $4 \pi / 30 \mathrm{rad} \mathrm{s}^{-1}$ over a period of approximately $6 \mathrm{~s}$. Dyed fluid from the more dense lower layer, when brought into the light sheet, results in a bright region in the flow visualization images.

The sequence of images shows the initial state (with some fluorescence from residual dye levels), followed by a number of bright concentric rings as dyed fluid is forced into the light sheet by the Ekman transport (directed towards the apex) impinging on the density interface. At $t^{*} \approx 30 \mathrm{~s}$, the structures formed from the more dense dyed fluid undergo a rapid breakdown, breaking the axisymmetry. The flow then evolves with an azimuthal periodicity until diffusing into the new state of a rigid body rotation.

\section{Conclusions}

This paper has discussed the linear spin-up of a two-layer density-stratified, rotating fluid in a variable-depth container. The container considered in the theoretical work consists of a finite-depth circular cylinder with a base formed from a cone cut horizontally near to the apex.

It has been shown that the readjustment mechanism in the lower layer remains qualitatively the same for any given relative position of the density interface and discontinuity in wall slope. However, careful consideration of the bounding viscous layers has also revealed an unusual (in comparison with classical spin-up problems) algebraic response in a sub-region of the upper layer when the density interface is below the cone/cylinder join.

A sequence of experiments has shown that, for sufficiently small changes in the 


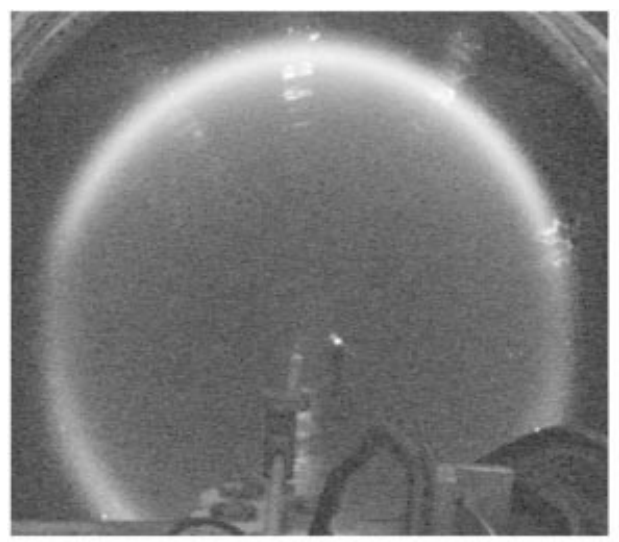

$t^{*}=0 \mathrm{~s}$

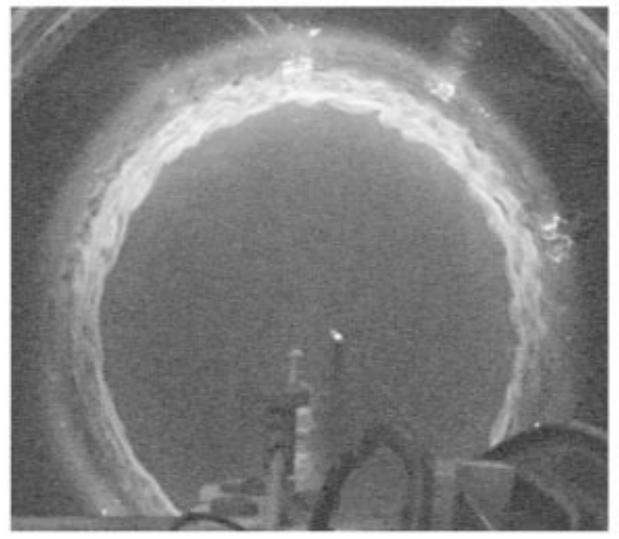

$t^{*}=30 \mathrm{~s}$

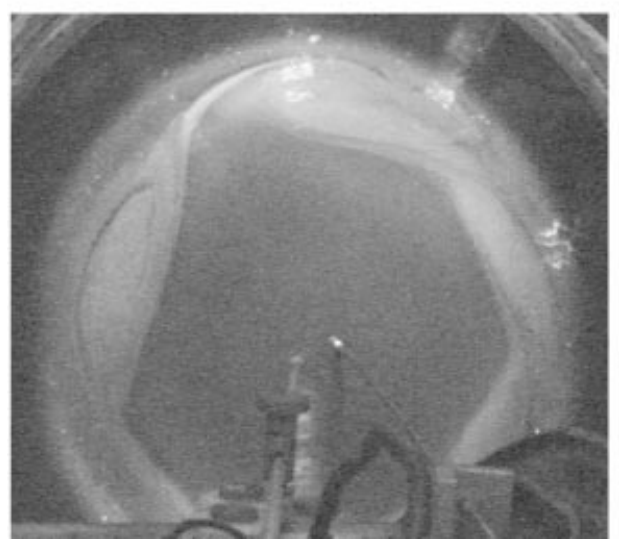

$t^{*}=120 \mathrm{~s}$

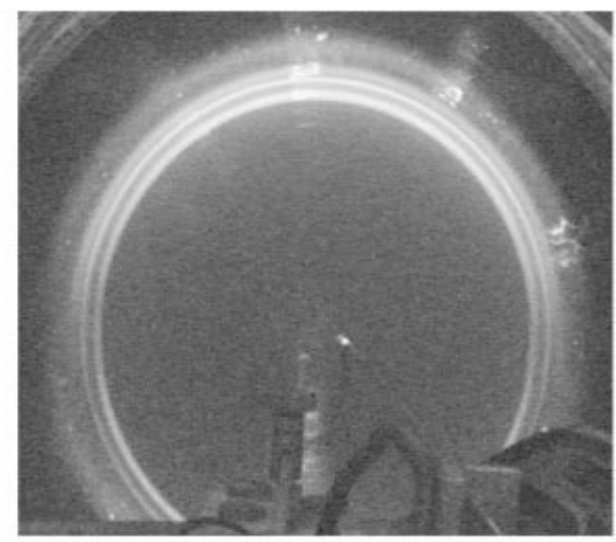

$t^{*}=12 \mathrm{~s}$

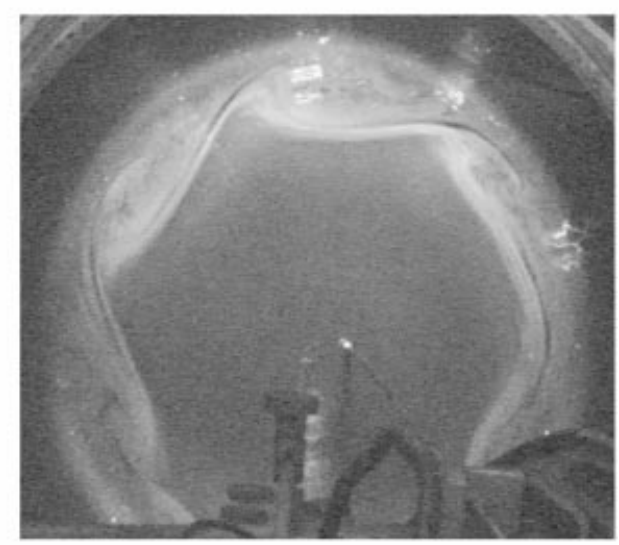

$t^{*}=80 \mathrm{~s}$

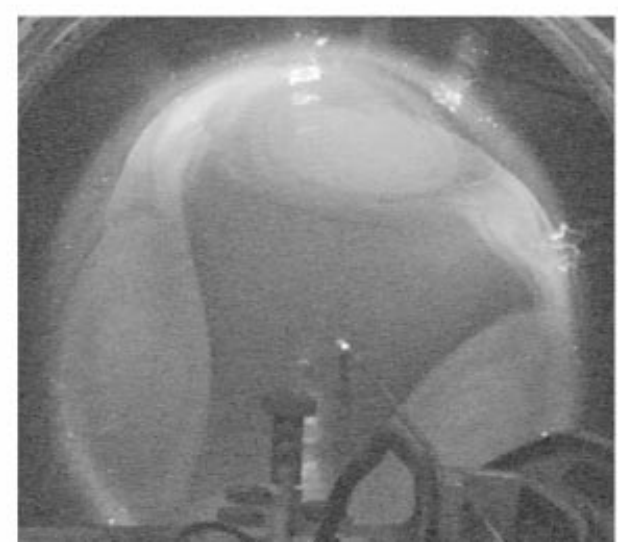

$t^{*}=200 \mathrm{~s}$

FIGURE 9. A sequence of video frames for experiment 6, showing the development of a non-axisymmetric flow near the density interface. The lower layer is dyed with fluoroscene and up-welling fluid from this layer intersects a horizontal light sheet to produce bright regions in the flow. 
angular frequency of the container, laboratory data are in good quantitative agreement with the theoretical predictions with regard to the readjustment mechanisms. The predicted diffusive response in the upper layer when the density interface is above the cone/cylinder join has not been confirmed quantitatively; however this difference arises over a time scale much longer than $E^{-1 / 2} \Omega^{-1}$. It is apparent that some slight recirculatory mechanism is active in this layer which can affect the experimental data over the longer time scale due to the absence of a dominant Ekman flow. Such a mechanism may arise from a transient doming of the interface/free surface, but is insufficient to affect comparisons in adjacent regions over the $E^{-1 / 2} \Omega^{-1}$ time scale.

A preliminary sequence of experiments has been performed for increasing values of the Rossby number to assess the first observed qualitative changes due to nonlinear effects. In this manner wave motion and non-axisymmetric flows in the near-interfacial region have been observed in the laboratory experiments. This unsteady non-axisymmetric flow regime has only been noted during nonlinear spin-down evolutions with the density interface intersecting the sloping base of the container. It is connected to an instability of the shear layer straddling $r=a$; however the vertical extent of the non-axisymmetric flow and its effect on the global spin-up time are subjects for future work.

The support of the EPSRC is gratefully acknowledged.

\section{Appendix}

It has been shown that the portion of Region II within $r=r_{B}$ spins up in the familiar way, with solution (2.23); furthermore, we found that in the portion of the fluid over the sloping bottom and under the interface, Region IIb, a physically similar spin-up occurs except that there is an unknown function $K(t)$ in equation (2.28). We utilize ideas due to Stewartson (1966) and Moore \& Saffman (1969b) to deduce the form of the function. $K$ is to be determined in the following way: From equation (2.28), we see that if $v$ were to be continuous at $r=r_{B}$, then there would be a net source of fluid at that location since the volumetric flow rates in the boundary layers are not identical due to the slope. That in itself is permissible since there can be a Stewartson 1/3-layer straddling the $r=r_{B}$ location, and such a layer can transport fluid that then passes out through the (thicker) 1/4-layers and back into the interior geostrophic flow. However, we know from Moore \& Saffman (1969b) that such a motion must be consistent with the possible solutions of the 1/3-layer. We now turn to the details of that analysis.

\section{A.1. Shear layer structure for the case $z_{c}<0$}

In a variety of places (see for example, Moore \& Saffman 1969b; Foster 1972), it is shown how to obtain the outer Stewartson layer (1/4-layer) equations, which are unsteady on the time scale considered here. We refer the reader to those sources, and simply note those equations here; they are

$$
\left.\begin{array}{l}
\frac{\partial v_{0 a}}{\partial t}-\frac{\partial V}{\partial t}+\frac{\partial^{2} V}{\partial \xi^{2}}=\frac{1}{h_{-}}\left(V-v_{0 a}\right), \text { for } \xi<0, \\
\frac{\partial v_{0 b}}{\partial t}-\frac{\partial V}{\partial t}+\frac{\partial^{2} V}{\partial \xi^{2}}=\frac{1}{h_{-}(\cos \alpha)^{1 / 2}}\left(V-v_{0 b}\right), \text { for } \xi>0,
\end{array}\right\}
$$




$$
\begin{aligned}
& \xi=\frac{\left(r-r_{B}\right)}{E^{1 / 4}} \\
& \mathscr{L}\{V\}=\mathscr{L}\left\{v_{0 a}\right\}+A \mathrm{e}^{p \xi}, \quad \xi<0, \\
& \left.\mathscr{L}\{V\}=\mathscr{L}\left\{v_{0 b}\right\}+B \mathrm{e}^{-q \xi}, \quad \xi>0,\right\} \\
& p \equiv\left(1 / h_{-}+s\right)^{1 / 2}, \quad q \equiv\left(\left([\cos \alpha]^{1 / 2} h_{-}\right)^{-1}+s\right)^{1 / 2} .
\end{aligned}
$$

Here the $0 a$ and $0 b$ subscripts refer to geostrophic velocities in Regions IIa and IIb evaluated at $r=r_{B}$. It is simplest to work, for the time being, with the Laplace transform of the 1/4-layer solutions.

In order to match the solutions to the interior 1/3-layer we introduce the 1/3-layer variable, $\eta=\left(r-r_{B}\right) / E^{1 / 3}$. Expanding solutions (A 2) for small $\xi$, then utilizing this $1 / 3$-layer scaling gives

$$
\begin{aligned}
& \mathscr{L}\{v\} \sim\left(\mathscr{L}\left\{v_{0 a}\right\}+A\right)+E^{1 / 12} A p \eta+\frac{1}{2} E^{1 / 6} A p^{2} \eta^{2}+\ldots \text { for } \eta \rightarrow-\infty, \\
& \mathscr{L}\{v\} \sim\left(\mathscr{L}\left\{v_{0 b}\right\}+B\right)-E^{1 / 12} B q \eta+\frac{1}{2} E^{1 / 6} B q^{2} \eta^{2}+\ldots \text { for } \eta \rightarrow \infty,
\end{aligned}
$$

which are matching conditions for 1/3-layer solutions. The 1/3-layers, which are steady on this time scale, are described by equations obtained from conventional boundary-layer arguments applied to (2.9)-(2.11), and are

$$
\begin{aligned}
& \frac{\partial^{3} v}{\partial \eta^{3}}+2 \frac{\partial w}{\partial z}=0, \\
& \frac{\partial^{3} w}{\partial \eta^{3}}-2 \frac{\partial v}{\partial z}=0, \\
& \frac{\partial u}{\partial \eta}+\frac{\partial w}{\partial z}=0 .
\end{aligned}
$$

We construct an asymptotic expansion for the solution of these equations in the form

$$
v \sim \bar{v}_{0}+E^{1 / 12} \bar{v}_{1}+E^{1 / 6} \bar{v}_{2}+\ldots
$$

Analysis of the solutions indicate (Moore \& Saffman 1969b) that the first two terms must be regular, and noting the form of (A 4)-(A 5) leads to solutions $v_{0}=f_{0}(t)$ and $v_{1}=f_{1}(t) \eta$. Matching to both 1/4-layers yields

$$
\begin{gathered}
\mathscr{L}\left\{\bar{v}_{0}\right\}=F_{0}(s)=\mathscr{L}\left\{v_{0 a}\right\}+A=\mathscr{L}\left\{v_{0 b}\right\}+B, \\
\mathscr{L}\left\{\bar{v}_{1}\right\}=F_{1}(s) \Rightarrow p A=-q B .
\end{gathered}
$$

Solving,

$$
A=\frac{q}{p+q}\left[\mathscr{L}\left\{v_{0}\right\}\right], \quad B=-\frac{p}{p+q}\left[\mathscr{L}\left\{v_{0}\right\}\right], \quad \text { where } \quad[\Psi] \equiv \Psi_{b}-\Psi_{a},
$$

so that $A$ and $B$ are now known in terms of the transforms of the geostrophic velocities evaluated at $r=r_{B} \pm$.

The third term in the (A 9) series satisfies equations (A 6)-(A 8), as do all terms. Integrating (A 6), written for $\left(\bar{v}_{2}, \bar{w}_{2}\right)$, over $z$ from $z=-h_{-}$to 0 gives

$$
\frac{\partial^{3}}{\partial \eta^{3}} \int_{-h}^{0} \bar{v}_{2} \mathrm{~d} z=\left.2 \bar{w}_{2}\right|_{-h_{-}} .
$$

Analysis of the solutions to (A 6)-(A 8) (see Moore \& Saffman 1969a) indicates that a source-like singularity at $\eta=0$ and $z=-h_{-}$is possible. Referring to figure 10 , 


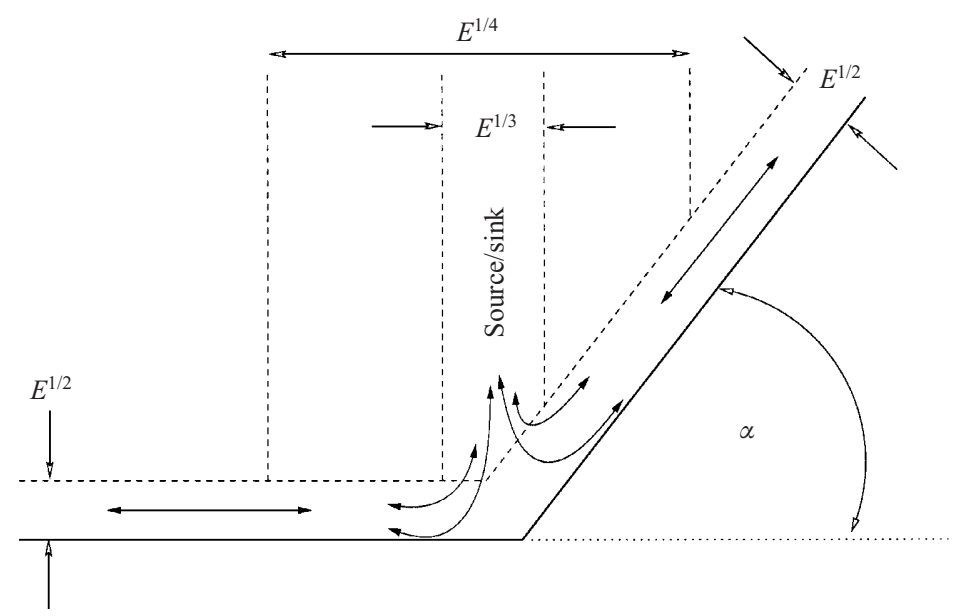

FIGURE 10. Source/sink behaviour in the Ekman layer at the discontinuity of wall slope.

fluid flows from the left, under the 1/4-layer, and fluid flows out to the right, under the right-hand 1/4-layer. There is a mismatch of this flux, and so equations (2.29) indicate that the Laplace transform of the net inflow of fluid into the base of the $1 / 3$-layer is

$$
\begin{gathered}
F_{\text {net }}=-\frac{1}{2} E^{1 / 2} \hat{F}_{n e t}, \\
\hat{F}_{n e t}=\left\{\left(\mathscr{L}\left\{v_{0 a}\right\}-\frac{v_{B}\left(r_{B}\right)}{s}\right)-\frac{\mathscr{L}\left\{v_{0 b}\right\}-v_{B}\left(r_{B}\right) / s}{(\cos \alpha)^{1 / 2}}\right\} .
\end{gathered}
$$

Since a source/sink-like singularity in $\left(\bar{v}_{2}, \bar{w}_{2}\right)$ is possible, as already noted, this net in/out-flow of fluid must be contained by the 1/3-layer, as shown in figure 10 . Therefore, on integrating (A 13) across the width of the 1/3-layer, we have a condition on the jump in the second derivative of $\bar{v}_{2}$, namely

$$
\frac{\partial^{2}}{\partial \eta^{2}} \int_{-h_{-}}^{0}\left[\mathscr{L}\left\{\bar{v}_{2}\right\}\right] \mathrm{d} z=\left.2 \int_{-\infty}^{\infty} \bar{w}_{2}\right|_{h_{-}} \mathrm{d} \eta=-\hat{F}_{n e t} .
$$

The integral on the left-hand-side is evaluated by the matching requirements given by the third term in the series (A 4), (A 5). Replacing $A$ and $B$ in that expression leads to the essential connection between the geostrophic velocities on either side of the shear layers and the Ekman inflow into the one-third layer,

$$
-p q h_{-}\left[\mathscr{L}\left\{v_{0}\right\}\right]=-\hat{F}_{n e t} .
$$

Substituting values for $p$ and $q$ and simplifying using $v_{0 a}$ as determined from (2.23) gives the Laplace transform of $v_{0 b}$ at $r=r_{B}$ to be

$$
\mathscr{L}\left\{v_{0 b}\right\}=\frac{v_{B}\left(r_{B}\right)}{s}-\frac{1}{p^{2}} \frac{1+p q h_{-}}{(\cos \alpha)^{-1 / 2}+p q h_{-}} v_{B}\left(r_{B}\right) .
$$

This result appears as equation (2.31) in the text.

\section{A.2. Shear layer structure for the case $z_{c}>0$}

In this case, the above analysis is applicable to the layers in $z<0$, so Region II spins up exactly as in the $z_{c}<0$ case. However, in $z>0$ there is a set of shear layers situated at $r=a$, where the interface contacts the sloping wall. Just to the right of the 
1/3-layer at $r=a$, the 1/4-layer is exactly as in Region II for $r=r_{B+}$; the governing equation is (A 2), but with $h_{-}$replaced by $h_{+}$. On the other hand, on $r=a_{-}$there is no Ekman layer either at $z=0$ or at $z=h_{+}$, so, referring to figure 10, there is no Ekman layer to the left of $\xi=0$, so there is no inflow to the 1/3-layer from there. Since there is no Ekman layer under the left-hand layer, it is not the usual 1/4-layer, that owes its structure to the existence of an underlying Ekman layer. In fact, the layer is simply diffusive. The shear layer equation is

$$
\frac{\partial v_{0 a}}{\partial t}-\frac{\partial V}{\partial t}+\frac{\partial^{2} V}{\partial \xi^{2}}=0 \text { for } \xi<0
$$

One can proceed as above, then, but apparently, on transforming (A 19), the quantity $p$ is now given by $s^{1 / 2}$ and $q$ by $\left(s+\mu / h_{+}\right)^{1 / 2}$. To distinguish from the previous section, let these values be denoted by $p^{\prime}$ and $q^{\prime}$. Since there is no interior spin-up in Region Ia on this time scale, and to leading order in $E, v_{0 a}=0$ and (A 10)-(A 11) leads to

$$
\mathscr{L}\left\{v_{0 b}\right\}=\frac{v_{B}(a)}{s} \frac{1}{1+p^{\prime} q^{\prime} h_{+}(\cos \alpha)^{1 / 2}} .
$$

However, the situation is in fact more subtle than indicated here. Unlike the other sub-regions discussed, this diffusive layer on $r=a_{-}$grows in width like $\sqrt{v t^{*}}$ in dimensional terms, or with a width $E^{1 / 4} t^{1 / 2}$ with the variables in use here. A more complete way of proceeding is to write down the solution to equation (2.24) inside Region Ia. The transform of that solution is

$$
\mathscr{L}\left\{v_{0}\right\}=\mathscr{K} I_{1}\left(\frac{\sqrt{s} r}{E^{1 / 4}}\right),
$$

where the constant $\mathscr{K}$ is not known, since its value comes out of the interaction at the layer. However, comparing this solution with the asymptotic expansion (A 4), we find after some algebra that

$$
p^{\prime}=\frac{E^{1 / 4}}{a} \frac{\psi\left(z^{2}+2\right)-1}{1-\psi}, \quad \psi \equiv \frac{1}{z} \frac{I_{1}(z)}{I_{0}(z)}=2 \sum_{n=1}^{\infty} \frac{1}{z^{2}+j_{0 n}^{2}}, \quad \text { where } \quad z \equiv \frac{\sqrt{s} a}{E^{1 / 4}} .
$$

In this expression, $j_{0 n}$ is the $n$th zero of $J_{0}$. So, now $\mathscr{L}\left\{v_{0 a}\right\}$ is no longer zero, and a modification to (A 20) arises, so that we have

$$
\begin{gathered}
\mathscr{L}\left\{v_{0 b}\right\}=\frac{v_{B}(a)}{s} \frac{1-Q}{1+p^{\prime} q^{\prime} h_{+}(\cos \alpha)^{1 / 2}-Q}, \\
\mathscr{L}\left\{v_{0 a}\right\}=-\frac{v_{B}(a)}{s} \frac{Q}{1+p^{\prime} q^{\prime} h_{+}(\cos \alpha)^{1 / 2}-Q},
\end{gathered}
$$

with

$$
Q \equiv \frac{q^{\prime}}{p^{\prime}+q^{\prime}} \frac{1-\left(z^{2}+1\right) \psi^{2}-\psi}{(1-\psi)^{2}} .
$$

So, (A 23) must in general replace (A 20). The Laplace inversion for $v_{0 b}$ and $v_{0 a}$ is indeed very complicated. Some general things may be noted, however. First, since the function $\psi$ is holomorphic, then so is the function $p^{\prime}$. Possible poles of $Q$ are accompanied by a branch point coming from $q^{\prime}$. It appears that the appearance of an origin branch point in $p^{\prime}$, incurred in writing $p^{\prime}=\sqrt{s}$ as above, is somehow an approximation to the actual inversion on a shorter time scale.

We now explore the nature of the Laplace inversion of these quantities. 
A.2.1. Long times

To examine the nature of the long-time features of the spin-up, we look at the neighbourhood of the origin of the s-plane. Recall that neither $p^{\prime}$ or $Q$ has a branch point at the origin! From examining the details, we find that

$$
\begin{gathered}
\psi \sim \frac{1}{2}\left(1-\frac{z^{2}}{8}+\frac{z^{4}}{48}+\ldots\right) \text { for } z \rightarrow 0 \Longrightarrow \\
p^{\prime} \sim \frac{3}{4} \frac{s a}{E^{1 / 4}}+O\left(s^{2}\right), \\
Q \sim 1-\frac{3}{4} z^{2}\left(1+\frac{E^{1 / 4}}{a q^{\prime}}\right)+O\left(z^{4}\right) .
\end{gathered}
$$

Using these results, it is trivial to obtain the residue of the origin poles for $\mathscr{L}\left\{v_{0 b}\right\}$ and $\mathscr{L}\left\{v_{0 b}\right\}$; the former has a simple pole, the second a second-order pole at $s=0$. Thus,

$$
\begin{aligned}
& \operatorname{Res}\left(\mathscr{L}\left\{v_{0 b}\right\} ; 0\right)=a\left(1-\frac{E^{1 / 4}}{a} \sqrt{\frac{\mu}{h_{+}}}\right), \\
& \operatorname{Res}\left(\mathscr{L}\left\{v_{0 a}\right\} ; 0\right)=\frac{4}{3 a} E^{1 / 2} t+O\left(E^{3 / 4} t\right) .
\end{aligned}
$$

So, it is interesting to note that even on the 'long' time scales analysed here, the interior of the fluid, in Region I, is only barely beginning to spin.

\section{A.2.2. Short times}

On the time scale primarily analysed in this paper, and the one relevant to the experimental measurements, $t=O(1)$, so the Laplace variable is also $O(1)$. That means that the quantities $p^{\prime}$ and $Q$ may be approximated for $z$ large. Doing that, we find that

$$
\begin{gathered}
\psi \sim \frac{1}{z}-\frac{1}{2 z^{2}}-\frac{1}{8 z^{3}}+\ldots \quad \text { for } \quad z \rightarrow \infty \Longrightarrow \\
p^{\prime} \sim \sqrt{s}-\frac{1}{2} \frac{E^{1 / 4}}{a}+O\left(\frac{E^{1 / 2}}{s}\right), \\
Q \sim-\frac{1}{2} \frac{q^{\prime}}{q^{\prime}+p^{\prime}} \frac{1}{z^{2}}+O\left(\frac{1}{z^{3}}\right),
\end{gathered}
$$

so that the result given above for $p^{\prime}$ actually represents the first term in a series. The details of the Laplace inversion are given more particularly in $\S 2.3$, but note here that in this range $\mathscr{L}\left\{v_{0 b}\right\}$ still has a simple pole at the origin, and $\mathscr{L}\left\{v_{0 b}\right\}$ on these shorter times still has a second-order pole. Hence,

$$
\begin{gathered}
\operatorname{Res}\left(\mathscr{L}\left\{v_{0 b}\right\} ; 0\right)=a, \\
\operatorname{Res}\left(\mathscr{L}\left\{v_{0 a}\right\} ; 0\right)=\frac{1}{2} E^{1 / 2} t+O\left(E^{3 / 4} t\right) .
\end{gathered}
$$

\section{REFERENCES}

Beardsley, R. C., Saunders, K. D. Warn-Varnas, A. C. \& Harding, J. M. 1979 An experimental and numerical study of the secular spin-up of a thermally-stratified rotating fluid. J. Fluid Mech. 93, 161.

Benton, E. R. \& Clark, A. 1974 Spin up. Ann. Rev. Fluid Mech. 6, 257. 
Buzyna, G. \& Veronis, G. 1971 Spin up of a stratified fluid: Theory and experiment. J. Fluid Mech. 50, 579.

Dalziel, S. B. 1992 Decay of rotating turbulence - some particle tracking experiments. Appl. Sci Res. 49, 217.

Duck, P. W., Foster, M. R. \& HewitT, R. E. 1997 On the boundary layer arising in the spin-up of a rotating stratified fluid in a container with sloping walls. J. Fluid Mech. 335, 233.

Foster, M. R. 1972 The flow caused by the differential rotation of a right circular cylindrical depression in one of two rapidly rotating parallel planes. J. Fluid Mech. 53, 647.

Greenspan, H. P. 1968 The Theory of Rotating Fluids. Cambridge University Press.

Greenspan, H. P. \& Howard, L. N. 1963 On a time-dependent motion of a rotating fluid. J. Fluid Mech. 17, 385.

Greenspan, H. P. \& Weinbaum, S. 1965 On nonlinear spin-up of a rotating fluid. J. Fluid Mech. 44, 66.

Heijst, G. J. F. van, Davies, P. A. \& Davis, R. G. 1990 Spin up in a rectangular container. Phys. Fluids A 2, 150.

Heijst, G. J. F. van, Maas, L. R. M. \& Williams, C. W. M. 1994 The spin-up of fluid in a rectangular container with a sloping bottom. J. Fluid Mech. 265, 125.

Hewitt, R. E., Davies, P. A., Duck, P. W. \& Foster, M.R. 1999a Spin-up of stratified rotating flows at large Schmidt number: experiment and theory. J. Fluid Mech. 389, 169.

Hewitt, R. E, Duck, P. W. \& Foster, M. R. $1999 b$ Steady boundary-layer solutions for a swirling stratified fluid in a rotating cone. J. Fluid Mech. 384, 339.

Hide, R. \& HockING, L. 1979 On detached shear layers and western boundary currents in a rotating homogeneous liquid. Geophys. Astrophys. Fluid Dyn. 14, 19.

KIM, K. Y. \& HyUn, J. M. 1994 Spin-up from rest of a 2-layer liquid in a cylinder. Trans. ASME: J. Fluids Engng 4, 808.

MacCready, P. \& Rhines, P. B. 1991 Buoyant inhibition of Ekman transport on a slope and its effect on stratified spin up. J. Fluid Mech. 223, 631.

Moore, D. W. \& SAfFman, P. G. $1969 a$ The structure of the free vertical shear layers in a rotating fluid and the motion produced by a rising body. Phil. Trans. R. Soc. Lond. A 264, 597.

Moore, D. W. \& Saffman, P. G. $1969 b$ The flow induced by the transverse motion of a thin disk in its own plane through a contained rapidly rotating viscous liquid. J. Fluid Mech. 39, 831.

O’DONNELl, J. \& Linden P. F. 1991 Free surface effects on the spin-up of fluid in a rotating cylinder. J. Fluid Mech. 232, 439.

Page, M. A. 1982 A numerical study of detached shear layers in a rotating sliced cylinder. Geophys. Astrophys. Fluid Dyn. 22, 51.

Pedlosky, J. 1967 The spin up of a stratified fluid. J. Fluid Mech. 28, 463.

Pedlosky, J. 1971 Geophysical fluid dynamics. In Mathematical Problems in the Geophysical Sciences (ed. W. H. Reid) Lectures in Applied Mathematics vol. 113, Amer. Math. Soc., Providence, RI, USA.

Phillips, O. M. 1970 On flows induced by diffusion in a stably-stratified fluid. Deep Sea Res. 17, 435.

SAKURAI, T. 1969 Spin down problems of rotating stratified fluid in thermally-insulated circular cylinders. J. Fluid Mech. 37, 689.

Spence, G. S. M., Foster, M. R. \& DAvies, P. A. 1992 The transient response of a rotating stratified fluid to impulsive surface forcing. J. Fluid Mech. 243, 33.

Stewartson, K. 1966 On almost rigid rotations. Part 2. J. Fluid Mech. 26, 131

Thorpe, S. A. 1987 Current and temperature variability on the continental slope. Phil. Trans. R. Soc. Lond. A 323, 471.

WALIN, G. 1969 Some aspects of time-dependent motion of a stratified rotating fluid. J. Fluid Mech. 36, 289.

Wedemeyer, E. H. 1964 The unsteady flow with a spinning cylinder. J. Fluid Mech. 20, 383.

Weidman, P. D. $1976 a$ On the spin up and spin down of a rotating fluid. Part 1. J. Fluid Mech. 77, 685.

Weidman, P. D. $1976 b$ On the spin up and spin down of a rotating fluid. Part 2. J. Fluid, Mech. 77, 709.

Wunsch, C. 1970 On oceanic boundary mixing. Deep Sea Res. 17, 93. 